\title{
Human Amniotic Fluid Mesenchymal Stromal cells (AF-MSCs) are Less Prone to in vitro Senescence Process Compared with Bone Marrow MSCs
}

\author{
Nicola Alessio', Caterina Pipino ${ }^{2}$, Domitilla Mandatori ${ }^{2}$, Pamela Di Tomo ${ }^{2}$, Angela Ferone', Marco Marchisio ${ }^{2}$, Paolo De Coppi ${ }^{3}$, Assunta Pandolfi ${ }^{2}$ and \\ Umberto Galderisi'
}

'Department of Experimental Medicine, Second University of Naples, Italy

${ }^{2}$ Department of Medical, Oral and Biotechnological Sciences, "G. d'Annunzio" University Chieti-Pescara, Chieti, Italy

${ }^{3}$ Surgery Unit, UCL Institute of Child Health, London, United Kingdom

Mesenchymal stromal cells (MSCs) are considered as an excellent source in regenerative medicine [1]. MSCs are of particular interest because they are being tested using cell and gene therapies for a number of human diseases. MSCs represent a rare population in tissues, for this reason they require, before being transplanted, an in vitro amplification. This process may induce replicative senescence, thus affecting differentiation and proliferative capacities.

Senescence is defined as the progressive and generalized impairment of function of the cells, resulting in an increasing vulnerability to environmental challenges and a growing risk of disease and death in an organism.

The purpose of this study was to investigate the in vitro behavior of MSCs from human amniotic fluid (AF-MSCs) and evaluate their senescence process. Indeed, it is know that amniotic fluid contains a population of MSCs easy to isolate, with great proliferative capacity, low immunological reactivity and low risk of graft-versus-host disease when transplanted. Moreover, AF-MSCs retain a normal karyotype and do not display tumorogenic potential even after extensive expansion in culture, thus resulting a great source of MSCs for the application in regenerative medicine.

AF-MSCs showed many features of bone marrow MSCs (BM-MSCs). Indeed, AF-MSCs had the surface antigens CD73, CD90 and CD105, which are the expression markers of BM-MSCs [2] and were able to differentiate into adipocytes, chondrocytes and osteocytes. Following this preliminary characterization, cells were monitored at different passages (P4, P7, P14) to evaluate the levels of senescence by $\beta$-galactosidase assay, length of telomeres, and analysis of the main pathways involved in the senescence (RB-P16, P53, P21). AF-MSCs evidenced a progressive increment of replicative senescence with increased expression of RB2, P16 and P27. Our data further evidenced that RB2 and not RB play a key role in MSC senescent process.

Interestingly, our results clearly evidenced that MSCs isolated from amniotic fluid are less prone to senescence process compared with bone marrow MSCs. Indeed, the senescence level of P4 bone marrow MSCs is reached only at P14 by AF-MSCs. These results may have a great therapeutic impact since AF-MSCs may be expanded in vitro for several passages without less of stemness and functionality.

\section{References}

1. Ikebe C, Suzuki K (2014) Mesenchymal stem cells for regenerative therapy: optimization of cell preparation protocols. Biomed Res Int.

2. Dominici M, Le Blanc K, Mueller I, Slaper-Cortenbach I, Marini F, et al. (2006) Minimal criteria for defining multipotent mesenchymal stromal cells. The International Society for Cellular Therapy position statement. Cytotherapy 8:315-317. 


\section{Immortalization of Human Adipose-Derived Stromal Cells as Possible Strategy to Employ their Paracrine Characteristics in Regenerative Therapies}

\section{Luigi Balducci}

Medestea Research \& Production laboratories, Consorzio Carso, Bari, Italy

Introduction: Human adipose-derived stromal cells (hASCs) are feasibly isolated and expanded. In addition, it is well established that they secrete large amounts of growth factors. Several findings suggest that, rather than trans-differentiation, hASCs could exert a therapeutic potential through a potent paracrine mechanism. All these features make hASCs a real and powerful therapeutic tool for the treatment of several human diseases. However, hASCs limited culture life-span may obstacle full employment of their paracrine characteristics in regenerative therapies. Under this perspective, hASCs immortalization may be a possible strategy to circumvent this problem.

Methods: hASCs were immortalized by co-transducing human telomerase reverse transcriptase (hTERT) gene with either SV-40 or human papilloma virus (HPV) E6/E7 genes. Flow cytometry was assessed to analyze mesenchymal marker expression. Differentiation potential was evaluated by immunocytochemistry and the levels of two cytokines, HGF and VEGF, were assayed by ELISA. Finally, the behavior of immortalized hASCs in basal medium was also investigated.

Results: Both hTERT/SV40 (TS) and hTERT/HPV E6/E7 (TE) co-transductions successfully immortalized hASCs. hASCs-TS and hASCs-TE reached elevated Population Doubling Levels (up to 100) and were expanded to obtain elevated cell number. Flow cytometry showed that both hASCs-TS and hASCs-TE retained a mesenchymal phenotypic profile, while differentiation potential decreased particularly in hASCs-TS. Of note, the levels of HGF and VEGF secreted by hASCs-TS and hASCs-TE remained elevated after immortalization. Finally, both hASCs-TS and hASCs-TE were successfully cultured in basal medium without FBS and/or exogenous recombinant proteins.

Conclusions: We have developed a "hybridoma-like model" that, by coupling hASCs immortalization and their paracrine characteristics, may have potential application in discovering and producing molecules to use in regenerative medicine (process scale-up). Moreover, such model may be very useful to eliminate direct injection of cells, thus avoiding potential adverse effects. 


\author{
Hydroxytyrosol Improves Function of Circulating Endothelial Progenitor Cells: Involvement of \\ Endothelial Nitric Oxide Synthase Activity and Expression \\ Carluccio MA ${ }^{1}$, Calabriso $N^{1}$, Scoditti E', Pellegrino $M^{1}$, Massaro $M^{1}$, Tinelli $A^{2}$, Storelli $C^{3}$ and De Caterina $R^{4}$ \\ 'National Research Council, Institute of Clinical Physiology, Lecce, Italy \\ ${ }^{2}$ Operative Unity of Gynecology and Obstetrics, Hospital "Vito Fazzi", Lecce, Italy \\ ${ }^{3}$ Department of Biological and Environmental Science and Technology, University of Salento, Lecce, Italy \\ ${ }^{4}$ G. d'Annunzio University, Chieti, Italy
}

Circulating endothelial progenitor cells (EPC) have been extensively studied as biomarkers to assess the risk of cardiovascular disease and as potential cell therapeutics for vascular regeneration [1]. EPC are exposed to oxidative stress during vascular injury, and there is a link between oxidative injury, endothelial cell dysfunction, and vascular disease. Endothelial dysfunction is characterized by a reduction of the bioavailability of vasodilators, particularly nitric oxide, and can predict future cardiovascular events [2].

Although many epidemiological studies have indicated that consumption of olive oil rich in polyphenolic compounds can reduce oxidative stress and improve endothelial function [3,4], the direct effect of olive oil polyphenols on the functions of EPC remains unclear. Aim of our research is to investigate the role of hydroxytyrosol, the major olive oil polyphenol, on the function of EPC and to evaluate the potential effect on endothelial nitric oxide synthase (eNOS) activity and expression.

EPC were isolated from human umbilical cord blood following the Hill method [5]. Mature endothelial cells were isolated from human umbilical vein (HUVEC) and cultured as previously described [6]. EPC and HUVEC were characterized for stem cells markers (CD133 and CD34) and endothelial markers (VE-cadherin and von Willebrand factor). We treated EPC and HUVEC with or without hydroxytyrosol (1-30 $\mu \mathrm{mol} / \mathrm{L}$ ) for $24 \mathrm{hr}$, after which we measured cell proliferation and in vitro angiogenesis using MTT and matrigel assay, respectively. Moreover the expression and activity of eNOS was evaluated by Western blotting. Hydroxytyrosol increased the number of EPC in a dose-dependent manner. Moreover hydroxytyrosol improved EPC and mature endothelial cell function through an enhancement of eNOS expression and activity. Finally, hydroxytyrosol ameliorated the EPC angiogenesis-like networks with HUVEC.

These findings highlight that hydroxytyrosol may improve the number and function of EPC and may contribute to olive oil cardiovascular protective effects

\title{
References
}

1. Vasa M, Fichtlscherer S, Aicher A, Adler K, Urbich C, et al. (2001) Number and migratory activity of circulating endothelial progenitor cells inversely correlate with risk factors for coronary artery disease. Circ Res. 89: 1-7.

2. Huang PH, Chen JW, Lu TM, Yu-An Ding P, Lin SJ (2007) Combined use of endothelial function assessed by brachial ultrasound and high-sensitive C-reactive protein in predicting cardiovascular events. Clin Cardiol. 30:135-140.

3. Covas MI, Nyyssonen K, Poulsen HE, Kaikkonen J, Zunft HJ, et al. (2006) The effect of polyphenols in olive oil on heart disease risk factors: a randomized trial. Ann Intern Med. 145: 333-341.

4. Moreno-Luna R, Munoz-Hernandez R, Miranda ML, Costa AF, Jimenez-Jimenez L, et al. (2012) Olive oil polyphenols decrease blood pressure and improve endothelial function in young women with mild hypertension. Am J Hypertens. 25:1299-1304.

5. Hill JM, Zalos G, Halcox JP, Schenke WH, Waclawiw MA (2003) Circulating endothelial progenitor cells, vascular function, and cardiovascular risk. N Engl J Med. 348:593-600

6. Carluccio MA, Siculella L, Ancora MA, Massaro M, Scoditti E et al. (2003) Olive Oil and Red Wine Antioxidant Polyphenols Inhibit Endothelial Activation : Antiatherogenic Properties of Mediterranean Diet Phytochemicals. Arterioscler Thromb Vasc Biol. 23: 622-629. 


\author{
Chemokine Stromal-Derived Factor 1 Alpha and Postoperative Septic Patients Undergoing Major \\ Abdominal Surgery in Urgency: A Pilot Study \\ Cotoia $A^{1}$, Cela $O^{2}$, Menga $M^{2}$, Stella $A^{1}$, Labella $D^{1}$, Liso $A^{1}$, Nazzareno $C^{2}$ and Cinnella $\mathbf{G}^{1}$ \\ 'Dipartimento Scienze mediche chirurgiche, Università Di Siena, Italy \\ ${ }^{2}$ Dipartimento di Medicina Clinica e Sperimentale, Università Di Siena, Italy
}

Background and Goal of Study: Septic shock is a systemic inflammatory syndrome to infection and it remains the most feared cause of death worldwide, with a high mortality rate in Intensive Care Units (ICU). Although the mechanisms that underline organ failure in sepsis have been partially elucidated, impaired cellular oxygen use plays a key role. The chemokine stromal-derived factor 1 alpha (SDF$1 \alpha$ ) is known to mediate the mobilization and migration of bone marrow-derived stem and progenitor cells in vivo. The underlying work hypothesis to test is that SDF-1a is involved in the septic shock as consequence of impaired tissue perfusion and cellular hypoxia.

Materials and Methods: In the prospective observational study were enrolled: 14 healthy volunteers (G1); 17 consecutive postoperative septic patients undergoing major abdominal surgery in urgency admitted in the ICU (G2); 9 non-septic patients undergoing surgical interventions (G3) at "Ospedali Riuniti”, Foggia.

Blood samples were collected on admission of the postoperative patients G2 and G3 in ICU and surgery ward (T0), at 24 hours (T1), and 3 (T3), 5 (T5) and 7 (T7) days postoperatively for quantitative analysis of SDF-1 $\alpha$ and pro-inflammatory interleukins (IL-1b, IL-2, IL-6, IL-8, IL-10, TNF, IL-4, IL-1a, VEGF, MCP-1, EGF). At any time were also collected the clinical parameters APACHE II and SOFA score, and the laboratory values of procalcitonin, C-reactive protein and endotoxin, white blood cells, red blood cells, hemoglobin and platelets. The data were evaluated by means of stepwise multivariate analysis.

Results and Discussion: The SDF-1 1 dosage showed a 50\% increase in the plasma of septic patients at admission as compared with either that of healthy subject or non-septic surgical patients $(74.5 \pm 18.5 \mathrm{pg} / \mathrm{ml}$ vs $48.9 \pm 7.4$ or $47.3 \pm 17.7)$. No significant changes in the SDF-1 $\alpha$ level was observed in G2 patients at the indicated post-operative interval times whereas a decrease was recorded in G3 patients at T3 and T5 followed by a recovery at T7. However, and interestingly, when the G2 patients were split in two subpopulations comprising surviving and deceased patients a 59\% increase of the SDF-1 $\alpha$ level was observed in the former while a $21 \%$ decrease was observed in the latter at T7. Normalization of the SDF-1a level measured at the different post-operative time points to the value measured at admission for each single patient (in order to reduce the interindividual variability) confirmed the trend observed on the average basis. However, the differences were not statistically significant because of the limited number of subjects. We also observed a positive correlation between the VEGF and SDF-1 1 levels at T1 and T2 in G2 (p=0.037).

Conclusion: The presented preliminary data indicate an increase of the circulating SDF-1 $\alpha$ level in the plasma of septic patients likely due to an adaptive response to impaired tissue perfusion and pseudo-hypoxia setting. Moreover it appears that patients capable of maintaining or even increasing the production of SDF-1 1 exhibit a better outcome following major abdominal surgery in urgency. This pilot study encourages the continuation of the research project on a larger sample size of patients to better understand if and how SDF-1 $\alpha$ is involved in the septic shock and its possible role in the recruitment/activation of endothelial progenitor cells or utilized as a prognostic biomarker 


\section{TRAIL-Engineered Umbilical Cord Mesenchymal Stem Cells (UC-MSC) as Cytotherapy Approach against Multiple Myeloma}

Morena d'Avenia, Annalisa Savonarola, Francesca Rizzo, Paola Cafforio and Francesco Silvestris

DIMO-Section, Clinical Oncology, University of Bari Aldo Moro, Italy

Background: Mesenchymal stem cells (MSCs) are currently under intensive investigation in experimental anti-cancer cell-based therapies and we have previously shown that umbilical cord (UC)-MSCs constitutively exert inhibitory activity on multiple myeloma (MM) cell growth both in vitro and in vivo in SCID mice as effect of their secretoma predominantly containing cytokines with suppressive properties on MM cell biology. In addition to this activity, UC-MSCs have been demonstrated to express chemotactic molecules as CXCR4 that activate their native property to migrate toward the tumor site and promote apoptosis in tumor cells by cell-to-cell cross-talk. Therefore, we considered UC-MSCs suitable for genetic modification to express TRAIL as major apoptogen molecule to be directly delivered to MM tumor cells.

Methods: MSCs from UCs were transduced by a bi-cistronic pMigr retroviral vector, expressing both GFP and TRAIL, under the control of the IL6 promoter. The choice to clone the IL6 promoter sequence before TRAIL was adopted to enhance the expression of the transgene in the presence of IL6 that is usually abundant within the MM tumor microenvironment and, thus, the membrane TRAIL isoform can be selectively expressed by TRAIL-transduced UC-MSCs in proximity of MM cells. Transduced cells were selected for GFP expression by sorting with FACS ARIA III, expanded in vitro, then assayed for GFP expression (85\% GFP ${ }^{+}$cells) and used for qRT-PCR studies, Western blot assays, ELISA test and co-cultures for functional studies. To evaluate the in vivo pIL6-TRAIL ${ }^{+} \mathrm{GFP}^{+}-\mathrm{UC}$-MSCs anti-myeloma activity, luminescent red-RPMI cells $\left(1 \times 10^{6}\right)$, generated by using RediFect ${ }^{\text {Tix }}$ Red-FLuc-Puromycin Lentiviral Particles, were intratibial injected in 4-6 weeks old SCID mice. After one week, the tumor growth was evaluated by relative luminescence using Lumina II Caliper, and pIL6-TRAIL $\mathrm{GFP}^{+}$-UC-MSCs, or control UC-MSCs, were locally or intravenously administered. Tumor burden was evaluated weekly by Lumina until 30 days after the MSCs treatment.

Results: After transduction, TRAIL mRNA levels were 40.000 fold higher in pIL6-TRAIL -GFP+-UC-MSCs in contrast with both intracellular and soluble amounts of TRAIL protein whose concentration was only 5 fold higher in transduced cells versus controls. Highest mRNA transcription occurred when MSCs were co-cultured with MM cell lines as both RPMI 8226 and U266. Moreover, functional data show that, after 48h and 72h, MM cells expressing on their surface DR4 molecules as major receptor for TRAIL, co-cultured with transduced pIL6-TRAIL ${ }^{+}-$GFP $^{+}-\mathrm{UC}-\mathrm{MSC}$ in trans-wells, underwent apoptosis at an extent at least double as compared to control UC-MSCs. This efficient anti-MM cytocidal activity was also confirmed in SCID mice intratibially transplanted with red-fluorescent MM cells developing bone tumor burden. In preliminary experiments, when these mice were intravenously administered with pIL6-TRAIL+-GFP ${ }^{+}$-UC-MSCs, an apparent shrinkage of the fluorescent bone tumor was recorded thus supporting the selective anti-MM activity of this cytotherapy approach.

Conclusions: Based on results obtained both in vitro and in vivo, our data support the potential approach to treat experimental MM in SCID mice with pIL6-TRAIL ${ }^{+}$-GFP ${ }^{+}$-UC-MSCs. Thus, in addition to the migratory property of UC-MSCs to the MM tumor site, TRAILtransduced cells are capable to reinforce their constitutive anti-MM activity and exert their pro-apoptotic effect by expressing TRAIL after the cross-talk with soluble factors of the MM tumor microenvironment. 


\section{Amniotic Stem Cells Biocompatibility on Electrospun Poly(Lactide-co-Glycolide) (PLGA), Poly( $\varepsilon$ - Caprolactone) (PCL), Poly(Lactic Acid) (PLA) Bioscaffolds after Different Sterilization Methods \\ Di Marcantonio L', Russo V ${ }^{1,5}$, Ancora $M^{2}$, Cammà $C^{2}$, Valbonetti L ${ }^{1,5}$, Turriani M1, Mauro A', Tammaro $L^{3,4}$ and Barboni B ${ }^{1,5}$}

'Facoltà di Medicina Veterinaria, Università di Teramo, Teramo, Italy

${ }^{2}$ Istituto Zooprofilattico Sperimentale dell'Abruzzo e del Molise "G. Caporale", Teramo, Italy

${ }^{3}$ Industrial Engineering Department, University of Salerno, Salerno, Italy

${ }^{4}$ ENEA- Brindisi Research Center, Brindisi- Italy;

${ }^{5}$ StemTech Group, Italy

Amniotic epithelial stem cells (AECs) retain great potential in cell-based regenerative medicine for their plasticity, immune tolerance and anti-inflammatory properties. Their performance in regenerative medicine would be increased by creating AECs engineered scaffolds allowing the regeneration of a tissue preserving its mechanical and structural properties. Prerequisite scaffolds must be sterilized prior to their preclinical/clinical use. Though, scaffolds sterilization is of particular concern, because sterilization methods can have significant effects on the structure of the used biomaterials and consequently on the biological effects on the seeded cells. Thus, in this study randomly oriented scaffolds of PLGA, PCL and PLA, biopolymers routinely used as medical devices, were firstly fabricated using electro-spinning process. Then, we investigated the effects of three different sterilization methods, ethanol (EtOH), ethanol rehydrated for 24 hours in culture media (EtOHr), and ethylene oxide (EtO), on scaffolds morphology. Finally, we analyzed the sterilized scaffolds biocompatibility for ovine AECs (oAECs). The analysis of scaffold structure with SEM showed a reduction in porosity in PLA after all sterilization methods, and in PLGA only by EtOH, while PCL got modified only by EtO causing microfibers complete fusion and loss of porosity. Successively, in order to evaluate the biocompatibility of oAECs for these biomaterials, ultrastructural and immunofluorescence analysis were performed on sterilized cell engineered scaffolds investigating cell engraftment, surviving proliferation index (PI), and DNA quantification. Ultrastructural analysis demonstrated oAECs engraftment in all scaffolds. Immunofluorescence confirmed a good cell integration and survival, although the type of biomaterial and its sterilization protocol influenced their distribution. In detail, oAECs seeded in PCL and in PLGA sterilized in EtOHr spread within the whole surface and especially around the microfibers. On the contrary, oAECs seeded in PCL sterilized in EtO did not spread over the entire scaffold surface but formed large cell clusters, probably due to the strong negative impact of the sterilization method on the scaffold structure. The influence of the different sterilization methods on scaffold structure affects also oAECs proliferation within the same type of biomaterial. In particular, oAECs PI for PLA scaffolds were significantly higher in EtOHr, for PCL scaffolds was higher in EtOH, and for PLGA scaffolds was higher in EtO compared to the other sterilization methods. DNA quantification demonstrated an increasing on cell population within the scaffolds compared to the initial quantity of DNA at the beginning of culture. The obtained results indicate that oAECs were able to colonize and to proliferate inside the analyzed electrospun biomaterials. Though, their biocompatibility grade and biologic activities are influenced by the scaffold sterilization method used. In conclusion, these oAECs engineered scaffolds could represent a good synergy and safe alternative in tissue regeneration, even if the design of the ideal scaffold should take in consideration several factors, such as, the sterilization method used and their potential preclinical/clinical application, prior allo- and xeno-transplantation settings. 


\author{
UC-MSCS Constitutively Exert Inhibitory Activity on Myeloma Cell Growth In vitro and In vivo \\ Claudia Felici, Morena d'Avenia, Valeria Simone, Paola Cafforio and Francesco Silvestris \\ DIMO-Section, Clinical Oncology, University of Bari Aldo Moro, Italy
}

Background: Mesenchymal stromal cells (MSCs) include stem cells with typical self-renewal and differentiation potential and are abundant in bone marrow (BM), in adipose tissue (AD-MSCs) and umbilical cord (UC), particularly in Wharton's jelly. MSCs have a controversial role in the pathogenesis of multiple myeloma (MM), since it has been alternatively ascribed to these cells either a supportive, or a suppressive activity on MM cell growth. In this context, recent studies suggest that UC-MSCs are resistant to genomic aberrations induced by MM cells on MSCs from other sources, while they exert a significant anti-tumor effect in vivo in a mouse lymphoma model. We investigated the effects of UC-MSCs on MM cell growth with respect to AD-MSCs as well as to normal and myelomatous BM-MSCs both in vitro and in SCID mice and explored relative molecular profiles.

Methods: The effect of AD-MSCs, normal BM-MSCs (n-BM-MSCs), MM-BM-MSCs and UC-MSCs on MM cell growth was assessed in co-culture by CFSE proliferation assay, whereas the clonogenic effect on MM cell growth by each MSC-conditioned medium (CM) was measured as number of colonies. The in vivo effect on MM tumor growth in presence of MSCs was evaluated in NOD-SCID mice, while differential content in cytokines and soluble factors in CMs was investigated by Multiplex ELISA and RNA microarrays.

Results: CFSE proliferation assay suggested that after $72 \mathrm{~h}$ of co-culture, MM cells maintained unaltered their proliferation extent both in presence of nBM-MSCs and MM-BM-MSCs, whereas underwent a significant decrease when cultured with either AD- or UC-MSCs ( $\mathrm{p}<0.05$ in both instances). Furthermore, the clonogenic assay showed that MM cells underwent three-fold decrease of the number of colonies by using AD and particularly UC-MSC CMs ( $\mathrm{p}<0.05)$. The in vivo experiments confirmed the anti-tumor activity of UC-MSCs. In particular, when RPMI-8226 cells were co-injected with UC-MSCs, NOD-SCID mice developed tumor masses $50 \%$ smaller than those treated with n-BM-MSCs.

Multiplex ELISA for 80 different proteins showed that, compared to UC-MSCs, both MM-BM and nBM-MSCs over-secreted nine factors involved in MM cell growth, namely IL-6, RANTES, EGF, FGF-4, IGF-1, IGFBP-1, IGFBP-2, IGFBP-6, and VEGF. On the contrary, UCMSCs prevalently produced cytokines with inhibitory activity as BMP-4, BMP-5, BMP-6, BMP-7, I-309, and TIMP-2. Finally, microarray assay revealed that genes of factors involved in MM biology were down-regulated in UC-MSCs in contrast with the high expression of genes for cell-to-cell communication, such as RALA, MPG, SMAD-4, OGT, GRB-2 and MAP3K7. Lastly, genes functionally implicated in the cell responses to inflammatory stimuli, such as $E L K-4, M C L-1, B C L-2$, and $M S H-2$, were significantly down-expressed, whereas $H G S, B C A R-1$, $P L O D-3$, and $P A F A H 1 B 1$, as genes related to the cell motility and chemotactic functions, were overexpressed up to three-fold in UC-MSCs as compared to nBM-MSCs.

Conclusion: Our data suggest that, in contrast with MSCs from other sources, UC-MSCs exert anti-MM activity and appear eligible for cell-based approach against this disease. Moreover, this native property needs to be better defined for future studies in cytotherapy models using engineered UC-MSCs to kill MM tumor cells. 


\section{CTR2 Identifies a Population of Cancer Cells with Stem-Like Features in Patients with Clear Cell Renal Cell Carcinoma}

Galleggiante $V^{1}$, Rutigliano $M^{1}$, Sallustio $F^{2,3}$, Ribatti $D^{3}$, Ditonno $P^{1}$, Bettocchi $C^{1}$, Selvaggi FP', Lucarelli $\mathbf{G}^{1}$ and Battaglia $M^{1}$

'Andrology and Kidney Transplantation Unit, Department of Emergency and Organ Transplantation-Urology, University of Bari, Bari, Italy

${ }^{2}$ Dialysis and Transplantation Unit, Department of Emergency and Organ Transplantation-Nephrology, University of Bari, Bari, Italy

${ }^{3}$ Department of Basic Medical Sciences, Neurosciences and Sensory Organs, University of Bari, Bari, Italy

Purpose: In clear cell renal cell carcinoma tissue samples we identified and characterized a population of renal cell carcinoma derived CD133+/CD24+ cancer cells. We studied differences between these cells and their non-neoplastic counterpart, tubular adult renal progenitor cells.

Materials and Methods: CD133+/CD24+ renal cell carcinoma derived cells were isolated from 40 patients. The mesenchymal phenotype and stemness proteomic profile of these renal cell carcinoma derived cells were characterized. Colony forming efficiency and self-renewal ability were tested by limiting dilution. Tumorigenic properties were evaluated in vitro by soft agar assay. The angiogenic response was evaluated in vivo by the chorioallantoic membrane angiogenic assay. Microarray analysis was performed on 6 tubular adult renal progenitor cell and 6 renal cell carcinoma derived cell clones. Membrane protein expression was evaluated by flow cytometry and immunofluorescence staining.

Results: CD133+/CD24+ cells were isolated from normal and tumor kidney tissue. Fluorescence activated cell sorting revealed that renal cell carcinoma derived cells did not express mesenchymal stem cell markers. CD133+/CD24+ tumor cells were more undifferentiated than tubular adult renal progenitor cells. Renal cell carcinoma derived cells were clonigenic and could differentiate into adipocytes, epithelial and osteogenic cells. They could also regenerate tumor cells in vitro and induce angiogenesis in vivo. Gene expression profile identified CTR2 as a membrane marker for this neoplastic population. CTR2 was involved in renal cell carcinoma derived cell cisplatin resistance.

Conclusions: Our results indicate the presence of a CD133+/CD24+/CTR ${ }^{2+}$ cancer cell population in clear cell renal cell carcinoma. These cells have some stem cell-like features, including in vitro self-maintenance and differentiating capabilities, and they can induce an angiogenic response in vivo. 


\author{
Exendin-4 Counteracts Palmitate-Induced Apoptosis and Autophagy in Human Cardiac \\ Progenitor Cells by Inhibiting De novo Production of Ceramide and p38 MAPK Phosphorylation

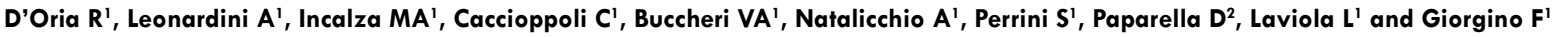 \\ 'Endocrinology, D.E.T.O, University of Bari Aldo Moro, Bari, Italy \\ ${ }^{2}$ Cardiac Surgery, D.E.T.O, University of Bari Aldo Moro, Bari, Italy
}

The incidence and prevalence of obesity- and diabetes-related co-morbidities are increasing in westernized countries. Lipotoxicity may contribute to the development of cardiac dysfunction associated with obesity and diabetes. The viability and responsiveness of cardiac progenitor cells is thought to be essential for constant tissue repair and renewal in the adult heart. Saturated fatty acids have been shown to induce premature aging, loss of replicative growth and increased apoptosis and autophagy in multiple cell types. Multiple pathways have been proposed to mediate free fatty acid (FFA)-induced apoptosis and autophagy, including activation of the stress kinases and the production of ceramides. However, the effects of palmitate on apoptosis and autophagy in human cardiac progenitor cells (hCPCs) have not been investigated. Analogs of the enteric hormone Glucagon-like peptide-1 (GLP-1) have recently emerged as effective glucose lowering agents. In addition, GLP-1 and GLP-1 analogs have been shown to exert prosurvival effects in multiple cellular models, including cardiac cells.

Therefore, the aim of this study was to investigate the mechanisms of fatty acid-mediated cell damage and the protective effects of the GLP-1 receptor (GLP-1R) agonist exendin-4 on palmitate-induced apoptosis and autophagy in hCPCs. hCPCs were isolated from right auricle biopsies of patients undergoing elective heart surgery and exposed to 0.1 to $0.5 \mathrm{mM}$ palmitate up to $24 \mathrm{~h}$. Dose- and timedependent increase of hCPC apoptosis was demonstrated by the evaluation of caspase- 3 activation, caspase- 3 cleavage, cytosolic levels of oligosomes $(\mathrm{p}<0.05)$ and by the TUNEL assay. In hCPCs exposed to $0.25 \mathrm{mM}$ palmitate for $16 \mathrm{~h}$ intracellular ceramide content, evaluated by immunofluorescence, resulted to be significantly augmented, in parallel with increased gene and protein expression of ceramide synthase 5 (CerS5), a critical enzyme in ceramide generation in the heart. Incubation of hCPCs with palmitate was also associated with increased autophagy, evidenced by autophagolysosome labeling, and confirmed by increased levels of the autophagic markers microtubule-associated protein 1 light chain 3 (LC3)-II and beclin $1(\mathrm{p}<0.05)$. Palmitate exposure also resulted in increased $\mathrm{p} 38$ MAPK phosphorylation ( $<0.05)$, whereas pretreatment with the p38 MAPK inhibitors SB203580 and SB202190 significantly inhibited palmitate-induced autophagy. Coincubation of hCPCs with fumonisin-B1, a specific CerS5 inhibitor, or CerS5 knockdown by a siRNA-based strategy partially prevented palmitate-induced apoptosis and autophagy $(\mathrm{p}<0,05)$, but had no effect on palmitate-induced p38 MAPK phosphorylation.

When hCPCs were pretreated with the GLP-1 analog exendin-4 (20nM for $8 \mathrm{~h}$ ), palmitate-induced apoptosis resulted to be prevented $(\mathrm{p}<0,05)$ and so was the increase in LC3-II and beclin1 protein expression $(\mathrm{p}<0.05)$ and in intracellular autophagosome labelling. The modulatory effects of exendin- 4 were abolished when cells were co-incubated with the GLP-1 receptor (GLP-1R) antagonist extending (939), or by inhibiting the GLP-1R signaling pathway with the PKA inhibitor H89, or by selectively reducing GLP-1R expression with specific siRNAs. Furthermore, exendin-4 prevented the increase of ceramide synthase 5 expressions, both at the gene and protein levels, and the increase of $\mathrm{p} 38$ MAPK phopshorylation that occurred in response to palmitate.

In conclusion, exposure to the GLP-1 analog exendin-4 prevents palmitate-induced apoptosis and autophagy in cardiac precursors isolated from human heart biopsies via inhibition of multiple signaling pathways. These results may contribute to explain the reported ability of GLP-1 and its analogues to counteract lipotoxic damage and to produce prosurvival effects on the human heart. 


\section{Reduced SIRT1 and SIRT2 Expression in Visceral Adipose Stem Cells may Promote Visceral Fat Expansion in Human Obesity}

Nigro $\mathbf{P}^{1}$, Porro $S^{1}$, Cignarelli $A^{1}$, Ficarella $R^{1}$, Capuano ${ }^{2}$, Puglisi $F^{2}$, Natalicchio $A^{1}$, Laviola $L^{1}$, Perrini $S^{1}$ and $G^{\prime}$,orgino $F^{1}$

'Endocrinology, D.E.T.O, University of Bari Aldo Moro, Bari, Italy

${ }^{2}$ General Surgery and Liver Transplantation, D.E.T.O. University of Bari Aldo Moro, Bari, Italy

Adipose tissue expands as a consequence of hypertrophy of preexisting fat cells and hyperplasia by recruitment of adipose stem cells (ASC) into the adipogenic program. SIRT1 and SIRT2 have been involved in regulation of adipocyte differentiation in rodent cells. In this study, we investigated the role of SIRT1 and SIRT2 in human adipocyte differentiation in obesity. Subcutaneous (Sc)- and visceral (V)-ASC were isolated from fat biopsies of 30 obese $(\mathrm{Ob})$ and 30 non-obese $(\mathrm{n}-\mathrm{Ob})$ donors and differentiated in vitro. Cell cultures were analyzed by immunofluorescence with Nile Red/DAPI to quantify triglyceride-containing cells (adipogenesis) and Oil-Red-O staining followed by spectrophotometry to measure accumulated triglycerides (lipogenesis). No differences in either adipogenesis or lipogenesis were found in Sc-ASC from $\mathrm{Ob}$ vs. $\mathrm{n}$-Ob donors. By contrast, both the number of triglyceride-containing cells and extent of triglyceride accumulation were 2 2.0-fold higher in $\mathrm{V}$-ASC from $\mathrm{Ob}$ compared to $\mathrm{n}$-Ob donors $(\mathrm{p}<0.05)$. These cellular phenotypes correlated with changes in SIRT1 and SIRT2 mRNA and protein levels, which were not different in Sc-ASC from n-Ob and Ob, and 50\% lower in V-ASC from Ob compared to $\mathrm{n}-\mathrm{Ob}(\mathrm{p}<0.05)$ subjects. Noteworthy, the selective overexpression of SIRT1 or SIRT2 in Ob V-ASC by adenoviral-mediated gene transfer resulted in down regulation of PPAR $\gamma$ and SREBP $1 \mathrm{c}(\mathrm{p}<0.05)$, and a significant $45 \%$ decrease in both lipogenesis and adipogenesis $(\mathrm{p}<0.05)$. Finally, assessment of SIRT1 and SIRT2 mRNA levels in human Sc and V fat biopsies from 149 donors with a wide BMI range showed a significant negative relationship between SIRT1/SIRT2 expression in V fat and BMI, both in males $(\mathrm{R}=-0.448, \mathrm{p}<0.05)$ and females $(\mathrm{R}$ $=-0.535, \mathrm{p}<0.05)$, while no correlation was found between SIRT1/SIRT2 expression in Sc fat and BMI. Thus, reduced SIRT1 and SIRT2 expression in V-ASC in human obesity may promote enhanced lipogenesis and adipogenesis leading to expansion of V fat. 


\section{Polychromatic Flow Cytometry Analysis to Identify and Subtype Circulating Microvesicles in Human Whole Peripheral Blood}

Marchisio $\mathrm{M}^{1-3}$, Lanuti $\mathrm{P}^{1-3}$, Ercolino $\mathrm{E}^{1,2 *}$, Simeone $\mathrm{P}^{1,2}$, Pierdomenico $\mathrm{L}^{1-3}$, Bologna $\mathrm{G}^{1,2}$ and Miscia $\mathrm{S}^{1-31}$

'Department of Medicine and Aging Science, School of Medicine and Health Sciences, University "G. d'Annunzio" Chieti-Pescara, Chieti 66100, Italy

${ }^{2}$ Center for Ageing Sciences (Ce.S.I.), “Università G. d'Annunzio" Foundation, Chieti 66100, Italy

${ }^{3}$ StemTeCh Group, Chieti 66100, Italy

Microvesicles (MVs) are small vesicles released by virtually all cell types and characterized by an integral plasma membrane, expressing the phenotype of their parental cells. MV carries relevant bio-actions and plays a crucial role in a multitude of pathologies (i.e. cardiovascular diseases, diabetes and atherosclerosis). Circulating MVs are increasingly thought to represent a potentially useful biomarker and polychromatic flow cytometry (PFC) is definitely the best tool to investigate them. Unfortunately, consensus guidelines on MV identification have not been reached so far, mostly due to the small size of these particles. Aim of the present study was to identify, characterize and count MV from untouched whole blood by a no-lyse/no-wash method, combined with MV volumetric count, based on a novel six-colour flow cytometry panel, so to identify and enumerate both the whole MV compartment and different MV subpopulations (endothelium-, platelet- and leukocyte- derived MV). Several panel combinations, anticoagulants and storage conditions were compared to set the best protocol in order to obtain reliable and reproducible MV counts. Results demonstrated that the application of the newly optimized PFC protocol here described and allowed to distinguish MV from exosomes, apoptotic bodies and other non-MV particles. This also obtains highly reproducible MV enumeration, pointing out the differences in terms of MV numbers and phenotypes between healthy donors, subjects at risk to develop cardiovascular events and cardiovascular disease patients. Finally, we also demonstrated that MV carries active mitochondria. These findings might open new routes for the monitoring of MV numbers, phenotypes and functions in different clinical settings. 


\section{Mapping the Human Amniotic Membrane: Useful Instructions for the Selection of Cells in Regenerative Medicine Approaches \\ Passaretta $\mathrm{F}^{1,2}$, Centurione $\mathrm{L}^{1,2}$, Centurione $\mathrm{MA}^{2,3}$, De Munari $\mathrm{S}^{4}$, Parolini $\mathrm{O}^{4}$ and Di Pietro $\mathrm{R}^{1,2}$}

'Department of Medicine and Ageing Sciences, Section of Human Morphology, G. d'Annunzio University of Chieti Pescara, Italy

${ }^{2}$ Ageing Research Center, Ce.S.I., G d'Annunzio University Foundation, StemTeCh Group, Chieti, Italy

${ }^{3}$ Institute of Molecular Genetics, National Research Council, Section of Chieti, Italy

${ }^{4}$ E. Menni Research Center, Poliambulanza Foundation, Brescia, Italy

The placenta is an important organ during pregnancy for the maintenance of feto-maternal tolerance, which is removed and discarded after childbirth. In recent years, there has been a growing interest in stem cells from the placenta, since it is a useful source of cells for the treatment of diseases in regenerative medicine without any ethical problems. In this study, we analyzed the amniotic membrane from the human placenta with the aim to map different regions with respect to the structure properties and regenerative potential. We dissected placentas of 6 healthy women $(31.16 \pm 6.3)$ that underwent a cesarean section at the hospital of Chieti as well as at the hospital of Brescia, to obtain samples for light and electron microscopy. In immunohistochemistry, we analyzed the expression of different markers of pluripotency and proliferation (OCT-4, c-KIT, SOX-2, $\alpha$-fetoprotein, CREB and p-CREB). Three areas were considered according to their position in relation to the umbilical cord, so that the first area was closer to the umbilical cord (the central area), the second and larger in the middle (the intermediate area), and the third was the most distant (the peripheral area). Morphometric analysis demonstrated that the OCT-4 and CREB expression in amniotic epithelial nuclei and cytoplasm was significantly higher in the peripheral area than in the intermediate area, whereas the central area had the weakest expression. Moreover, both the c-KIT and SOX-2 expression in amniotic epithelial cells, as well as p-CREB expression, were higher in the peripheral area, while $\alpha$-fetoprotein expression was significantly higher in the central area. Similar results were obtained in amniotic mesoderm with respect to OCT- 4 and $\alpha$-fetoprotein expression, whereas no differences were found between the three areas in SOX-2 expression. Interestingly, the amniotic epithelium from both the central area and the peripheral area was occasionally multi-layered and displayed detaching cells or cell cytoplasm fragments, indicating the existence of amniotic membranederived exosomes. In consistence with these observations, electron microscopy analysis displayed a greater presence of lipid granules in epithelial cells from the peripheral area of amniotic membrane.

Our morphological and histo-chemical analysis highly suggests that the different areas of amniotic membrane are characterized by different cells types that could present a different regenerative potential. Functional assays are being performed to confirm our preliminary observations with the aim to increase efficiency of amniotic membrane engraftment within a therapeutic context. 


\section{Establishment of 3D-Dynamic Osteoblasts-Osteoclasts Co-Culture Model to Simulate the Jawbone Microenvironment in vitro}

Penolazzi L, Lolli A, Sardelli L, Angelozzi M, Ciarpella F, Vecchiatini R, Lambertini E and Piva R

Department of Biomedical and Specialty Surgical Sciences, University of Ferrara

The research on bone diseases highlights the need for reliable experimental models that may faithfully recapitulate in vitro the pathological bone microenvironment, and therefore provide a valuable tool for the development of novel strategies for bone regeneration. Taking into account that, at its simplest level, the production of bone mineralized tissue requires the presence of osteoblasts (hOBs) and osteoclasts (hOCs) enclosed in a structured matrix, nowadays much effort is focused on the set-up of specific in vitro hOBs/hOCs co-culture systems. Such simplified experimental models allow a functional characterization of the single cell types, while preserving the intimate crosstalk that naturally occurs in vivo between bone-forming and bone-resorbing cells. Our work was aimed at establishing a 3D hOBs/hOCs co-culture model to simulate the microenvironment present either in healthy or pathological jawbone, specifically for osteopenic or osteonecrotic diseases. In particular, we focused on bisphosphonate-related osteonecrosis of the jaw (BRONJ), a clinical complication found in patients treated with bisphosphonates leading to impaired jawbone turnover and tissue necrosis. We first confirmed the possibility to isolate hOBs from samples of necrotic bone (hnOB) obtained by BRONJ patients, despite the poor quality of the biological specimens. hnOB cultured in vitro maintained the typical features of osteoblastic cells, such as expression of osteogenic markers (OPN, Runx2) and mineralization capacity after culture in osteogenic medium. For the set-up of the co-culture model, our choice fell on mature and osteoprogenitor cells namely hOBs from nasal septum and MSCs from periodontal ligament (hPDLMSCs), respectively. By co-culturing hOBs or hPDLMSCs with monocytes from peripheral blood (hMCs) in transwell plates, we first demonstrated their ability to induce osteoclasts (hOCs) maturation in the absence of inducers after 7 days of co-culture. Culture in osteogenic medium for further 14 days induced the expression of OPN and deposition of mineral matrix, confirming that the co-culture system preserved the functional activity of the hOBs. We then established and compared two $3 \mathrm{D}$ co-culture systems, carried out in static condition using agarose-coated wells (3D-C) or dynamic condition using the Rotary Cell Culture System (3D-Dyc). After optimization of the culture parameters, we determined the lowest cell numbers that could allow the formation of viable hOBs/hOCs constructs, as the low amount of cells is a major issue when working with osteonecrotic samples. We demonstrated the presence of mature hOCs in the constructs cultured in 3D already after 7 days, in the absence of osteoclastogenic inducers. After further 14 days of culture in osteogenic medium, constructs derived from both 3D-C and 3D-Dyc culture systems were highly viable and showed the presence of mature hOCs and bone mineral matrix within the aggregates. In addition, the constructs stained positive for OPN and Col1A1. However, constructs cultured in 3D-C condition were poorly structured and showed areas with a disorganized matrix, while constructs cultured in 3D-Dyc had a very well definite structure with a uniform distribution of the mineral matrix. In conclusion, the formation of viable constructs is possible also co-culturing limited amounts of hOBs and hMCs in 3D-Dyc condition in the absence of osteoclastogenic inducers and avoiding the use of exogenous scaffolds. The finding that it is possible to obtain functional cells from anatomic locations with less than adequate bone quality and volume or a compromised area lacking sufficient stem and progenitor cells due to disabling conditions such as BRONJ, and ii.to create reliable 3D combinations with a few cells opens new scenarios to achieve autologous implantable constructs. 


\title{
Osteogenic Differentiation of Human MSCs: Specific Occupancy of the Mitochondrial DNA by NFATc1 Transcription Factor
}

\author{
Angelozzi $\mathbf{M}^{1}$, Ciarpella $\mathrm{F}^{\mathbf{1}}$, Penolazzi L', Lambertini $\mathrm{E}^{1}$, Lolli $\mathrm{A}^{1}$, Lisignoli $\mathbf{G}^{2}$, Pinton $\mathbf{P}^{3}$ and Piva $\mathbf{R}^{1}$ \\ 'Department of Biomedical and Specialty Surgical Sciences, University of Ferrara, Ferrara, Italy \\ ${ }^{2}$ Laboratorio di Immunoreumatologia e Rigenerazione Tissutale, IOR, Bologna, Italy \\ ${ }^{3}$ Department of Morphology, Surgery, Experimental Medicine University of Ferrara, Ferrara, Italy
}

Variations of number, structure, function and intracellular distribution of mitochondria are correlated with cell functionality and different cell energy demands. These variations, which are strictly associated with a finely tuned crosstalk between the mitochondrial and nuclear genomes, have recently been recognized as essential events during the differentiation process of stem cells and cell fate switch. Efforts to uncover the mechanisms of mitochondrial biogenesis as well as to characterize energy metabolism and redox status during cell differentiation have been made, but little is known about mitochondria transcription regulation by lineage-specific factors and signaling demands. In particular, molecular regulatory circuits that govern mitochondrial dynamics together with mitochondrial contribution to differentiation potential of stem cells remain poorly understood. Hence, exploring the properties of mitochondria during differentiation of cellular progenitors is important in gaining information on stem cell biology, and developing new pharmacologic strategies in the field of regenerative medicine. In addition, this may facilitate the understanding of maintenance of cell culture homeostasis and the optimization of in vitro cell differentiation protocols by adjusting some biochemical properties, such as energy production or redox status of mitochondria. These improvements could provide high quality stem cells to be used for cell therapy. In this scenario, mitochondrial properties could be used as a quality measure of cell-based products for several clinical uses.

Recent studies demonstrate that mitochondria are maintained at a low activity state in human mesenchymal stem cells (hMSCs). Upon osteogenic induction, their functions increase to fulfill a higher degree of energy demand or to facilitate other biochemical reactions that take place within the organelles. However, the high energy aerobic demand by osteoblasts at the early stages of differentiation, is necessarily slowed down during the progress of calcified matrix deposition and at the end of differentiation when the cells become apoptotic or quiescent. The regulation of these dynamics has still to be studied but it is reasonable to hypothesize that specific signals are sent from the nucleus to mitochondria to change their activities.

Among the molecules that influence the decision of hMSCs to become osteoblasts there is NFATc1 transcription factor. NFATc1, through calcineurin and calmodulin, is implicated in the regulation of gene expression by calcium signaling, the control of which involves the mitochondria. We demonstrated for the first time that NFATc1 is localized in the mitochondria. Using chromatin immune-precipitation assay, we found that NFATc1 is recruited in vivo at the non-coding displacement loop (D-loop) regulatory region of mtDNA, but this occurs only when the calcification process is at its highest in osteo-induced hMSC and the maximum level of differentiation is reached. Occupancy of the mtDNA by NFATcl is associated with a decreased expression of crucial mitochondrial genes such as Cytochrome B and NADH dehydrogenase 1 . This suggests that NFATc1 acts as a negative regulator of mtDNA transcription during the calcification process and supports interruption of aerobic energy demand when it is no longer needed.

The finding of NFATc1 participation in terminal osteogenic differentiation through its direct involvement in the regulatory machinery of mitochondria suggests a new role for this transcription factor and adds information on communication between mitochondrial and nuclear genomes. 


\title{
Regenerative Medicine
}

\section{Large-Scale Generation of Highly Purified Human iPSC-Derived Neurons to Establish a Human Cell Model for Neurotropic Viruses}

\author{
D'Aiuto $L^{1}$, Nimgaonka VL ${ }^{1,2}$ and Viggiano $L^{3}$ \\ 'Department of Psychiatry, Western Psychiatric Institute and Clinic, University of Pittsburgh School of Medicine, Pittsburgh, USA \\ ${ }^{2}$ Department of Human Genetics, Graduate School of Public Health, University of Pittsburgh, Pittsburgh, USA \\ ${ }^{3}$ Department of Biology, University of Bari "Aldo Moro", Bari, Italy
}

iPSC technology offers an unprecedented opportunity to study human neurological diseases enabling new approaches to understanding their mechanisms. The ability to generate and manipulate large number of nervous cells bridges the gap between studies using animal models and human clinical research.

We developed a protocol for large-scale generation of neuronal stem cells (NSCs)/early neural progenitor cells (eNPCs) and their differentiation into neurons from iPS cell lines. Targeted mass spectrometry analysis demonstrates that iPSC-derived neurons express ligand-gated channels and other synaptic proteins and whole-cell patch-clamp experiments indicate that these channels are functional. We exploited this protocol to pave the way for understanding the species specific pathogenic effects of neurotropic viruses, such as HSV-1 and HCMV that has been limited by difficulties in sustaining primary human neuronal cultures. We derived iPS cells from human adult fibroblasts and induced neural lineages to investigate their susceptibility to infection with HSV-1. Though currently available treatments (e.g., acyclovir) are effective in limiting the duration and severity of recurrent infections, latent infection is incurable, thus providing a lifelong reservoir of infection in humans. Most of our knowledge regarding the molecular mechanisms of HSV-1 latency comes from rodents models (mice and rabbits) that are not the natural hosts for HSV-1. Even if animal models have importance in understanding latency and persistence of HSV-1 infection, observed differences in the regulation of HSV-1 latency among mice and rabbit models and even between mouse strains suggest that different regulatory mechanism could be present in latently infected human neurons.

This led us to believe that the establishment of a human cell model can enable a direct understanding of the molecular mechanisms of latency in our species. Moreover, this approach will let us to perform high-throughput screening of novel drugs that will be able to definitively eradicate HSV-1 from the sensory ganglia neurons (such as trigeminal ganglia). 


\title{
Epigenetics Modification of Male Germ-Like Cells Derived From Human Amniotic Fluid Stem Cells
}

\author{
Antonucci $I^{1,2}$, Provenzano $M^{1}$, Rodrigues $M^{1}$, Alfonsi $M^{3}$, Sorino $L^{1}$, Ballerini $P^{1}$, Palka $G^{3}$ and Stuppia $L^{1,2}$ \\ 'Department of Psychological, Health and Territorial Sciences, Laboratory of Molecular Genetics, School of Medicine and Health Sciences, G. d' Annunzio University, Chieti-Pescara, Italy \\ ${ }^{2}$ Stem Tech Group, Center for Aging Studies (CESI), Chieti, Italy \\ ${ }^{3}$ Department of Clinical, Oral and Biotechnological Sciences, School of Medicine and Health Sciences, G. d' Annunzio University, Chieti-Pescara, Italy.
}

Several studies have proposed that in vitro generation of germ cells from stem cells can be considered as an excellent model in vitro for elucidating the molecular mechanisms of gametogenesis. Errors in germ cell development cause infertility, and mutations carried by germ cells lead to disorders in the offspring. In the last years, it has been reported in the literature that human Amniotic Fluid-derived Stem Cells (hAFSCs) have the ability to express specific markers of primordial germ cells (PGCs), and in particular genes involved in early stages of germ cell development. Due to these remarkable features, hAFSCs show characteristics similar to pluripotent cells of the epiblast and therefore could be considered ideal candidates to generate in vitro germ cells. The purpose of this particular study was to investigate the epigenetic modifications during differentiation of hAFSCs into male germ-like cells. hAFSCs were cultured in suspension in uncoated petri dishes for EBs formation and at day 4 Retinoic Acid (RA) or Testosterone (T) or RA and T in combination was added into the culture medium. During the initial two days of induction, EBs were positive for alkaline phosphatase (AP) staining and round cells along with a small number of spermatogonia-like cells were observed in the treated cultures. Quantitative PCR was performed to characterize differentiated cells from EBs and the expression analysis demonstrated that cells treated were able to express early germ cell markers (Fragilis, Stella, Vasa, Piwil Dazl, c-Kit, RNH2), meiotic gene (Boll) and some post-meiotic germ cell markers (PRM-1, PRM-2, GPX4, PGK2). Genomic DNA was isolated simultaneously from EBs at day 3, day 7, day 10 and day 14 of induction and thereafter global DNA methylation status was examined. The methylation experiments have demonstrated a gradual demethylation of DNA in cell derived from EBs treated with RA between day 10 and day 14 of differentiation. Methylation of the CpG islands of imprinted gene H19 was investigated by bisulfite sequencing. Complete demethylation was detected in EBs at day 3 of differentiation and high levels of remethylation were observed at day 14, indicating de novo methylation by this stage. The dynamic demethylation of the paternally methylated H19 was observed in our experiments which are in agreement with the data reported in the literature. In conclusion, the results obtained suggest that hAFSCs have the potential to differentiate into male germ cells and in the future could be used in disease modelling of infertility to study mutagenic and epigenetic effects of drugs. 


\section{Mesenchymal Stem Cells Nanovesicles: Innovative Therapeutic Approach for Neuroprotection and Neuroregeneration}

\section{Bruno Bonetti}

Department of Neurological and Movement Sciences, University of Verona, Verona, Italy

The efficacy of mesenchymal adipose-stem cells (ASC) has been demonstrated in different neurodegenerative and neuroinflammatory animal models, although concerns about their safety have been raised, particularly related to their persistence in ectopical niches. For this purpose, we have tested the efficacy of nanovesicles (NV) from ASC in different experimental models of neurological diseases. We found that NV increased the cell viability of neuroblastoma cell line $\mathrm{SH}_{-} \mathrm{SY} 5 \mathrm{Y}$ and of primary hippocampal neurons exposed to $\mathrm{H}_{2} \mathrm{O}_{2}$ by reducing the apoptotic rate. We also tested the neuroregenerative potential in the ex vivo model of cerebellar slice cultures incubated with the demyelinating agent lysophosphatidylcholine. We found that NV induced a 2.2 fold increase of precursor cells by immune blotting and a parallel 5 fold increase of $\mathrm{MBP}^{+}$myelinated areas by immunohistochemistry as compared to control. We finally verified the efficacy of NV in the MOG-induced EAE model in vivo by injecting e.v. at days 3, 5, 8 post-immunization. We found that NV significantly ameliorated the clinical course with marked decrease of inflammatory and demyelinating scores examined at disease peak. Interestingly, the proliferation and the cytokine profile in vitro of MOG-specific T cells were significantly modulated by NV.

Taken together, these results suggest that ASC-NV may represent a new therapeutic approach for neurological diseases, being able to protect neuron, promote regenerative processes and modulate immune responses. 


\section{Identification of a Novel Cell Population Endowed with a Healing Capacity Present in the Peripheral Blood of Healthy Mice}

\section{Claudia Lo Sicco, Roberta Tasso, Daniele Reverberi, Michele Cilli, Ulrich Pfeffer and Ranieri Cancedda}

Universita' di Genova \& IRCCS AOU San Martino - IST, Istituto Nazionale per la Ricerca sul Cancro, Largo Rosanna Benzi 10, 16132, Genova, Italy

Since the end of the last century, major progresses have been made with regard to the transplant of "ex vivo" expanded autologous stem/progenitor cells, in most cases associated to a biomaterial carrier. However, with few exceptions, this therapeutic approach never really took-off. There are scientific aspects, such as vascularization of large size implants, identification of the "optimal" source of cells and the "optimal" biomaterial carrier that require further investigations. Moreover, additional bottlenecks are: i) the logistic of collecting from patients, expanding in culture and returning the cells to the surgical theater; ii) the high cost of the culture procedure within the GMP facilities required by the strict rules defined by National and European Regulatory Agencies.

Several decades of stem cell research have progressively uncovered within developed organs a stunning capacity for endogenous regeneration and repair, mediated by specialized stem and progenitor cells residing in their native constitutive tissues. We report on the identification of a rare population of cells present in the peripheral blood of healthy mice that actively participates in the tissue repair/ regeneration process. Injury signals are sufficient to (i) specifically direct the recruitment to the wound site of these Circulating Healing $(\mathrm{CH})$ cells; (ii) promote their differentiation and appropriate integration in the regenerative microenvironment. $\mathrm{CH}$ cells were identified by an innovative flow cytometry strategy as small cells not expressing CD45 and lineage markers. The analysis of their global transcriptome revealed their uniqueness when compared to other cells characterized by varying stemness degree. Moreover, $\mathrm{CH}$ cells presented a high expression of key pluripotency-associated genes and positive selective markers of the epiblast developmental stage. 


\title{
Long-Term Effect of Progesterone in Preservation of the Epithelioid Phenotype of Amniotic Epithelial Cells in vitro
}

\author{
Canciello $A^{1}$, Barboni $B^{1,2}$ and Parolini $\mathrm{O}^{3}$ \\ 'Department of Comparative Biomedical Science, University of Teramo, Teramo, Italy \\ ${ }^{2}$ Stem TeCh Group, Chieti, Italy \\ ${ }^{3}$ Centro di Ricerca E. Menni, Fondazione Poliambulanza - Istituto Ospedaliero, Brescia, Italy
}

Introduction: Epithelial-to-Mesenchymal Transition (EMT) is a transdifferentiation process whereby epithelial cells acquire a mesenchymal phenotype. EMT plays a crucial role in the regulation of different events such as embryogenesis, cancer progression and stem cells biology [1]. This phenomenon involves also Amniotic Epithelial Cells (AEC) during the in vitro expansion thus modifying the functional attitudes of this emerging source of pluripotent cells. When AEC are cultured, they turn spontaneously into stromal-like cells and start to express the mesenchymal markers (Vimentin and a-SMA) by losing the epithelial ones (Cytokeratin and E-Cadherin) [2]. The phenotype shift is, in parallel, accompanied by a dramatic reduction of AEC plasticity and immunomodulatory properties, both essential to justify their use in cell therapy [3]. But physiologically, AEC does not undergo EMT during the embryo/fetus development, when the cells are exposed to a well-defined environment characterized by low oxygen and high levels of steroids (primarily progesterone: $\mathrm{P}_{4}$ ), a condition that have not been yet adopted into the in vitro protocols.

Aim of the Study: The present research has been designed to verify whether $\mathrm{P}_{4}$ could prevent the spontaneous EMT in vitro by preserving AEC phenotype and genotype.

Material and Methods: Isolated AEC and whole amniotic membrane (AM) were collected at the slaughterhouse, isolated under validated protocol [4] and incubated in presence of different concentration of $\mathrm{P}_{4}$ (from $100 \mu \mathrm{M}$ to $0.01 \mu \mathrm{M}$ ) with or without its inhibitor (RU-486). AM were analysed in terms of morphology (Hematoxylin and Eosin staining) and cell viability (Calcein and Propidium live-dead kit) over 3 days of culture.

AEC were, in addition, evaluated for proliferative rate (doubling-time, DT), epithelial and mesenchymal markers expression (Cytokeratin 8 and $\alpha$-SMA, respectively) and pluripotency genes expression (Oct-3/4, Sox 2 and Nanog) over 4 passages.

Results and Conclusion: $\mathrm{AM}$ cultured in the presence of $25 \mu \mathrm{M} \mathrm{P}$ maintained unaltered their morphology and membrane integrity up to 3 days, in contrast with CTR ones. CTR AM, indeed, showed a greater incidence of cell death than $\mathrm{P}_{4}$-treated AM $(42.4 \pm 3.7 \% v s 13.6 \pm$ $4.3 \%$, respectively).

Similarly, the AEC incubated with $25 \mu \mathrm{M} \mathrm{P}_{4}$ maintained their epithelioid morphology and a higher positivity for Cytokeratin 8 over 4 cultural passages. By contrast, during in vitro expansion, untreated cells (CTR) acquired early a mesenchymal phenotype by increasing the incidence of $\alpha$-SMA positive cells. Furthermore, $\mathrm{P}_{4}$ decrease the DT of AEC respect to the CTR $(83.25 \mathrm{~h} v s 29 \mathrm{~h}$, respectively). The specificity of $\mathrm{P}_{4}$ in preventing the in vitro EMT has been further confirmed through the treatment with $\mathrm{P}_{4}$ inhibitor, RU-486. Indeed, AEC cultured simultaneously with $\mathrm{P}_{4}$ and RU-486 displayed a rapid epithelial phenotype shift similar to that recorded in CTR. Noteworthy, $\mathrm{P}_{4}$ increases the expression of Oct-3/4, Sox 2 and Nanog after the first passage. Taken together, these data demonstrate that $\mathrm{P}_{4}$-treated AEC maintain their epithelial phenotype for a long time in culture and simultaneously increase the expression of pluripotency markers. The possibility to preserve in vitro the AEC native properties may be quite useful to improve their use in cell-based regenerative protocols after long-term expansion.

Acknowledgment: This work was supported by Fondazione Tercas and PRIN 2010.

\section{References}

1. Guo F, Kerrigan BCK, Yang D, Hu L, Shmulevich I, et al. (2014) Post-transcriptional regulatory network of epithelial- to-mesenchymal and mesenchymal-toepithelial transitions. J Hematol Oncol, 7: 1-11.

2. Bilic G, Zeisberger SM, Mallik AS, Zimmermann R, Zisch AH (2008) Comparative characterization of cultured human term amnion epithelial and esenchymal stromal cells for application in cell therapy. Cell Transplant. 17: 955-968.

3. Stadler G, Hennerbichler S, Lindenmair A, Peterbauer A, Hofer K, et al. (2008) Phenotypic shift of human amniotic epithelial cells in culture is associated with reduced osteogenic differentiation in vitro. Cytotherapy. 10: 743-752.

4. Barboni B, Curini V, Russo V, Mauro A, Di Giacinto O, et al. (2012) Indirect Co-Culture with Tendons or Tenocytes Can Program Amniotic Epithelial Cells towards Stepwise Tenogenic Differentiation. PLoS One, 7: 1-14 


\section{Extracellular Vesicles: Paracrine Mediators of Stem Cell-Induced Tissue Regeneration \\ Vincenzo Cantaluppi, MD}

Nephrology, Dialysis and "A. Vercellone" Kidney Transplantation Center, University of Torino, "Città della Salute e della Scienza- Molinette" University Hospital, Torino, Italy, E-mail: vincenzo. cantaluppi@unito.it

Extracellular vesicles (EVs) are circular fragments of membrane released from the endosomal compartment as exosomes or shed from the surface membranes of most cell types. An increasing body of evidence indicates that EVs play a key role in cell-to-cell communication. Indeed, EVs may directly stimulate target cells by receptor-mediated interactions or may transfer from the cell of origin various bioactive molecules including membrane receptors, proteins, mRNAs and particularly microRNAs, small non coding RNAs of 20-22 bp able to modulate protein transduction in target cells.

The pleiotropic biologic effects of EVs are relevant for communication among cells in both physiological and pathological conditions including inflammation, sepsis, tumor progression and acute or chronic renal diseases. Recent evidences suggested the presence of a bidirectional exchange of genetic information between stem and injured cells. The transfer of gene products from injured cells may explain stem cell functional and phenotypic changes without the need of transdifferentiation into tissue cells. On the other hand, transfer of gene products from stem cells may reprogram injured cells to repair damaged tissues.

In this setting, several studies clearly demonstrated the regenerative potential of bone marrow-derived Mesenchymal Stromal Cells (MSCs) and Endothelial Progenitor Cells (EPCs) in experimental models of Acute Kidney Injury (AKI) and in the setting of kidney transplantation. EVs derived from both these stem cell types have been shown to participate in kidney regeneration after acute ischemic or nephrotoxic insults.

We herein show that after experimental kidney ischemia-reperfusion injury, EPC-derived EVs localized within peritubular capillaries and tubular epithelial cells. This conferred functional and morphologic protection from AKI by enhanced tubular cell proliferation, reduced apoptosis, and leukocyte infiltration. EPC EVs also protected against progression of chronic kidney damage by inhibiting capillary rarefaction, glomerulosclerosis, and tubulointerstitial fibrosis. The renoprotective effect of EVs was significantly reduced after their treatment with RNase or after depletion of specific mRNAs and/or microRNAs within EVs. Indeed, EPC-derived EVs carried anti-apoptotic, proangiogenic and complement inhibitor mRNAs and microRNAs that can contribute to reprogramming injured resident renal cells toward a regenerative program. 


\author{
Energetic Metabolism in Glioblastoma Stem Cells: Effects of PPARalpha Antagonists \\ Fidoamore $A^{1}$, Cristiano $L^{1}$, Benedetti E', D'Angelo $M^{1}$, Galzio $R^{1}$, Cinque $B^{1}$, Cifone $M G^{\prime}$, Giordano $A^{2,3}$ and Cimini $A^{1,3}$ \\ 'Department of Life, Health and Environmental Sciences, University of L'Aquila, Italy \\ ${ }^{2}$ Department of Medicine, Surgery \& Neurosciences, University of Siena, Italy \\ ${ }^{3}$ Sbarro Institute for Cancer Research and Molecular Medicine and Center for Biotechnology, Temple University, Philadelphia, PA, USA
}

Glioblastoma multiforme (GBM, grade IV astrocytoma) is the most malignant and aggressive of brain tumors with poor prognosis and a median survival of patients of approximately 15 months. Over the past decade there has been an increasing interest in the role of cancer stem cells in the tumorigenesis process, because they are responsible of tumor drug resistance and relapse. It is known that glioblastoma stem cells (GSCs) are a heterogeneous population that resides in the intratumoral perivascular and necrotic/hypoxic niches and that hypoxia exerted a crucial function, maintaining stemness and governing their fate. Particularly, hypoxia inducible factors (HIFs) regulate many aspects of tumor malignant progression such as proliferation, angiogenesis, metastasis and metabolism, whose mechanisms, in GSCs, today are poorly understood. In the brain Peroxisome proliferator activated Receptors (PPARs), ligand-activated transcription factors, belonging to the nuclear hormone receptor superfamily, play a specific role in the energetic metabolism. Three isotypes have been described, the $\alpha, \beta$ and $\gamma$, that have a tissue specific distribution and pleiotropic functions as they are involved in many ways in cell proliferation, differentiation, glucose and lipid metabolism.

In this study we report the presence of PPAR $\alpha$ in GSCs and, its pivotal role in controlling their proliferation and glucose and lipid metabolism, by the use of a specific antagonist, GW6471.

We isolated and characterized cancer stem cells (CSCs) from bioptic GBM specimens and evaluated the expression of PPARs ( $\alpha$ and $\gamma$ ) and proteins implicated in glucose and lipid metabolism under normoxia and hypoxia conditions, focusing on some proteins of peroxisomal and cholesterol pathways and glycogen and lipid storage. It is known that lipid droplets are important organelles in energy fuel and in intercellular signaling and we have previously demonstrated that their presence is correlated to the grade of the malignancy.

Our results show that in hypoxia, PPAR is upregulated and there is an increase of glycogen storage and lipid droplets. By using the PPARa antagonist in both conditions, we observed, a dramatic reduction of cell proliferation and of glycogen and lipid content, that was paralleled by an increase of glycogen demolition and a reduction of cholesterol synthesis and lipid transport. We propose therefore the use of specific PPARa antagonists, as adjuvant agent in standard therapy, to render GSCs nutrients-starved, acting subsequently on their proliferation rate and survival. 


\title{
Regenerative Medicine
}

\author{
Identification of Epigenetic Modifications in Liver Stem Cells Differentiation \\ Cozzolino $A M^{1}$, Noce $V^{1}$, Bisceglia $F^{1}$, Battistelli $C^{1}$, Santangelo $L^{1}$, Grassi $G^{2}$, Tripodi $M^{1,2}$ and Amicone $L^{1}$ \\ IIstituto Pasteur-Fondazione Cenci Bolognetti, Department of Cellular Biotechnologies and Haematology, Sapienza University of Rome, Italy \\ ${ }^{2}$ L.Spallanzani National Institute for Infectious Diseases, IRCCS, Rome, Italy
}

The current challenge of experimental hepatology is to develop new methods and protocols to overcome organ transplantation. Stem cells represent a crucial tool for setting up effective therapeutic approach based on liver tissue engineering.

To this end the dissection of molecular events driving liver stem cell maintenance and differentiation is crucial. In these processes an early and central role is played by epigenetic modifications, by which stemness genes are silenced and lineage-specific genes are activated [1].

We isolated, established in line and characterized a new resident liver stem cell, RLSC [2]; these cells show a "metastable" phenotype (i.e. co-expression of mesenchymal and epithelial markers) and the unique feature to differentiate both in epithelial (hepatocytes) and mesenchymal (stellate cells) liver cells, when orthotopically transplanted [3].

We investigated the epigenetic events anticipating the hepatocyte specific gene expression, and in particular, the chromatin modifications of the HNF4a promoter (the master transcriptional regulator of hepatic differentiation).

At this purpose we cultured RLSCs on hydrogels of acrylamide and bisacrylamide with modulus of elasticity of $0.4 \mathrm{kPa}$, comparable to normal liver stiffness; these culture conditions are able to induce a rapid modulation of molecular pathways of mechano-transduction and an early (within 24 hours) acquisition of hepatocyte-like morphology and specific gene expression. The differentiation onset correlates with a rapid histone modification of HNF4a promoter chromatin (H3K27me3, H3K9ac and H3K4me3) at the transcriptional activators HNF1/6 binding sites.

Conversely RLSCs grown on high stiffness substrates $(80 \mathrm{kPa})$ maintains stem cell properties.

In conclusion, i) we set up culture conditions, based on tunable stiffness of extracellular matrix, able to drive a rapid and efficient hepatocyte differentiation of RLSC or, on the contrary, their maintenance in an undifferentiated state; ii) we identified specific chromatin modifications on the promoter of the hepatocyte differentiation master gene HNF4a driving cellular choices at the branch of hepatic differentiation.

The knowledge of the epigenetic signatures necessary for specific transcription factors recruitment can be useful to manipulate stem cells (in vivo and in vitro) controlling their fate and therefore provide insights for the design of new therapeutic strategies.

\section{References}

1. Chen T, Dent SY (2014) Chromatin modifiers and remodellers: regulators of cellular differentiation. Nat Rev Genet. 15: 93-106.

2. Conigliaro A, Colletti M, Cicchini C, Guerra MT, Manfredini R, et al. (2008) Isolation and characterization of a murine resident liver stem cell. Cell Death Differ. 15:123-133.

3. Conigliaro A, Amicone L, Costa V, De Santis Puzzonia M, Mancone C, et al. (2013) Evidence for a common progenitor of epithelial and mesenchymal components of the liver. Cell Death Differ. 20:1116-1123. 


\section{Biomimetic Porous Titanium Scaffolds for Large Bone Critical Defect Reconstruction: An Experimental Study \\ Crovace $A M^{1}$, Lacitignola $L^{1}$, Monopoli-Forleo $D^{2}$, Santos-Ruiz $L^{4}$, Becerra $J^{4}$, Di Meo $A^{1}$, Francioso $E^{3}$ and $C r o v a c e ~ A^{3}$}

'Dottorato di Ricerca in Sanità e Scienze Sperimentali Veterinarie -DMV University of Perugia, Italy

${ }^{2}$ Biomedical Engineering Department, Canary Islands Institute of Technology, Canary Islands, Spain

${ }^{3}$ DETO, University of Bari "Aldo Moro", Italy

${ }^{4}$ BIONAND, Nanomedicine and Biotechnology Center of Andalucia, University of Málaga, Spain

The main goal of the treatment of large bone defect is directed to guarantee a precocious loading of the affected limb. In the present paper the authors propose a new technique to reconstruct by use of Biomimetic porous titanium scaffold [1] custom made with Electron Beam melting technology that evidenced to be suitable to reach the purpose. A complete resection was practiced in the diaphysis of the right tibia of six sheep and replaced with a five centimeters framework of EBM-sintered titanium. The outcome was followed-up by periodical X-ray and clinical investigations with a follow-up of 12 months. At 9 months the plates were removed, at 12 months the sheep were euthanized and the tibia were subjected to histological and immunohistochemical investigations. The post-operative X-ray showed a good position of the plate and the proximal and distal border of the scaffolds were perfectly adherent to the bone to permit the process of osteointegration. After surgery the sheep were allowed to move freely in the stables Nine months after the X-ray showed a remodelling of periostal callus with a well-defined cortical bone and the scaffolds were completely integrated in the diaphysis. The histological investigations were executed on bone-metal interface and showed bone growth among the titanium bars, bone trabeculae have bridged the titanium trabeculae suggested a very good tissue-metal interaction. In conclusion this implant, used to repair large bone critical defects in a large animal model, can guarantee the immediate body-bearing, a precocious functional recovery and a good osteointegration the non-stained thick section (1.5 $\mathrm{mm}$ ). The sections stained with von Kossa's suitable to observe calcified bone showed how, forming a metal-bone interconnected network with a histological pattern suggestive of bone remodelling. Bone trabeculae have been formed around the EBM-sintered titanium trabeculae creating an interconnected system and the tight joining among them suggested a very good tissue-metal interaction. The geometry of the porous implants seems to better promote osteointegration of the implant itself. In conclusion this kind of implant, used to repair large bone critical defects in a large animal model, can guarantee the desirable immediate body-bearing, a precocious functional recovery and a good osteointegration.

\section{References}

1. Mastrogiacomo M, Corsi A, Francioso E, Di Comite M, Monetti F, et al. (2006) Reconstruction of extensive long bone defects in sheep using resorbable bioceramics based on silicon stabilized tricalcium phosphate. Tissue Eng. 12: 1-13. 


\section{Potential Dual Role of Vitamin K2 in Reducing Calcium Deposit in Vascular Smooth Muscle Cells from Hypertensive Rats while Increasing Osteogenesis in Human Mesenchymal Stem Cells Derived from Amniotic Fluid}

Mandatori D', Pipino $C^{1}$, Di Pietro N' ${ }^{1}$ Di Tomo $\mathbf{P}^{1}$, Di Silvestre $\mathbf{S}^{1}$, Failli $\mathbf{P}^{2}$ and Pandolfi $A^{1}$

'University "G. d'Annunzio", Chieti-Pescara, Aging Research Center, Ce.S.I., "G. d'Annunzio" University Foundation, Chieti-Pescara, StemTeCh Group, 66013 Chieti, Italy

${ }^{2}$ University of Florence, Florence 50139, Italy

Cellular differentiation process in cardiovascular and bone biology may play a key role in "bone-vascular cross talk" both in physiological and pathological conditions, such as hypertension and osteoporosis. Recently, it has been shown that Vitamin K2 (VitK2), a lipid-soluble vitamin, plays a main role in this crosstalk by reducing the calcium deposit in the arteries and increasing it in the bone tissue ("calcium paradox"), through carboxylation and then activation of specific Gla proteins (Matrix Gla protein and osteocalcin respectively). Although recent data suggest that aortic smooth muscle cells (SMCs) from Spontaneously Hypertensive Rats (SHR) tend to become easily calcified, nothing is known about the potential protective role of VitK2 in this phenomenon. Moreover, since the mechanism of vascular calcification is similar to that of osteogenesis, it might be important to study the potential role of VitK2 also in the osteogenesis of human mesenchymal stem cells derived from amniotic fluid (hAFMSCs).

Thus, aims of our study were to investigate, in vitro, the possible role of VitK2 in the i) modulation of osteogenesis in a model of hAFMSCs differentiated in osteogenic cell; $i$ ) reduction of vascular calcification in a model of SMCs derived from thoracic aortas of SHR and their relative controls (WKY, Wistar Kyoto).

In order to evaluate its potential cytotoxicity, we tested in both cellular models the effect of VitK2 (0.5-10 $\mu \mathrm{M}, 7$ days) on cell proliferation/ vitality test (3- [4,5-dimethylthiazol-2-yl] - 2,5-diphenyltetrazolium bromide, MTT Assay). We found that VitK2 at any concentrations employed did not affect the cell vitality and proliferative ability in both cell cultures studied. Then we assessed the effect of VitK2 on hydroxyapatite precipitation through Alizarin Red S staining. In the first set of experiments, we added VitK2 to hAFMSCs at the same time of the osteo-differentiation induction. In these experimental conditions we did not observe any significant VitK2 effects on osteogenesis. Thus, in the second set of experiments, VitK2 was added on hAFMSCs previously exposed for 7 days to osteogenic medium, to allow the expression of osteocalcin. Interestingly, in these experimental conditions VitK2 (1, 2.5 and $5 \mu \mathrm{M}$ for 14 days) significantly increased hydroxyapatite deposites, compared to hAFMSCs treated with osteogenic medium alone. As expected, VitK2 at all doses used (2.5, 5 and 10 $\mu \mathrm{M})$ significantly reduced hydroxyapatite deposit in SMCs from SHR after 21 days' exposure to calcification medium. The same effect was observed in control cells after 14 and 21 days' exposure to VitK2. Taken together these data clearly suggest the dual role of VitK2 in reducing the calcification process in SMCs while improving it in hAFMSCs. Therefore, VitK2 may have an important role in the prevention of both cardiovascular and bone related diseases, thus encouraging further in depth studies to reach its use as natural food supplement.

Acknowledgment: This study was partially supported by Ibersan-Santiveri. 


\section{Osteogenic Differentiation of Equine Umbilical Cord Matrix Mesenchymal Stem Cells upon Stimulation with Calcium-Sensing Receptor (CaSR) Agonists \\ Martino NA' ${ }^{1}$, Reskhin SJ', Lacalandra $\mathrm{GM}^{2}$, Nicassio $\mathrm{M}^{2}$, Ciani $\mathrm{E}^{1}$ and Dell'Aquila $\mathrm{ME}^{1}$}

'Dipartimento Bioscienze, Biotecnologie e Biofarmaceutica (DBBB), Università di Bari, Bari, Italy

Dipartimento dell'Emergenza e Trapianti d'Organo (DETO), Università di Bari, Bari, Italy

Background: Fetal adnexa-derived mesenchymal stem cells (MSC) have a wide range of potential therapeutical applications. The scientific and clinical interest of equine MSC, as a model for stem cell-based therapy of orthopaedic injuries, is increasing. The extracellular calcium-sensing receptor (CaSR) plays a major role in calcium homeostasis and bone formation/resorbtion. We investigated the involvement of CaSR in triggering osteogenic differentiation of two equine size-sieved umbilical cord matrix (UCM) MSC lines, previously characterized, by reverse transcription PCR, as positive for MSC markers (CD105, CD44, CD29 and CD166) and negative for hematopoietic markers (CD34, CD14 and MHC-II). The effects of AMG641, a novel potent research type II calcimimetic acting as CaSR agonist, in presence of high extracellular calcium concentration $\left[\mathrm{Ca}^{2+}\right] \mathrm{o}$, were determined.

Methods: Umbilical cords $(n=3)$ were collected at the end of delivery from three mares hospitalized at the Veterinary Clinics of University of Bari for parturition assistance, according to standard veterinary practices. Large $(>8 \mu \mathrm{m}$ in diameter) and small $(<8 \mu \mathrm{m})$ equine UCMMSC lines were isolated from perivascular Wharton's jelly of equine UCM, cultured in osteogenic differentiation medium containing: 1) low $\left.\left[\mathrm{Ca}^{2+}\right]_{\mathrm{o}}(0.37 \mathrm{mM}) ; 2\right)$ high $\left[\mathrm{Ca}^{2+}\right]$ o $\left.(2.87 \mathrm{mM}) ; 3\right)$ the CaSR agonist AMG641 $(0.05,0.1$ or $1 \mu \mathrm{M})$ with high $\left[\mathrm{Ca}^{2+}\right]$ o and 4$)$ the CaSR antagonist NPS2390 (10mM for $30 \mathrm{~min})$ followed by incubation with AMG641 in presence of high $\left[\mathrm{Ca}^{2+]} \mathrm{o}\right.$. Expression of osteogenic differentiation molecular (Runt-related transcription factor, RUNX2 and Osteopontin, OPN as assessed by Real Time PCR) and histochemical (Alkaline phosphatase activity and calcium phosphate deposition, as assessed by Von Kossa staining) biomarkers were compared among groups.

Results: Elevating $\left[\mathrm{Ca}^{2+}\right]$ o during osteogenic differentiation in vitro culture significantly increased the relative abundance of osteogenic differentiation biomarkers compared with control. In the large cell line, additional $\mathrm{Ca}^{2+}$ up-regulated $\mathrm{OPN}(\mathrm{P}<0.05)$ and $\mathrm{CaSR}(\mathrm{P}<0.05)$ mRNA expression even if it had no effect on relative expression of RUNX2, whereas in the small cell line, high [Ca $\left.{ }^{2+}\right]$ o increased both OPN and RUNX2 transcripts level $(\mathrm{P}<0.001$ and $\mathrm{P}<0.01$, respectively) but it had no effects on CaSR expression. Osteogenic molecular markers expression was differentially regulated by AMG641, with stimulatory effects (OPN up-regulation) in the large cell line (up to $\mathrm{P}<0.01$ ) and inhibitory effects (RUNX2 and OPN down-regulation) in the small cell line (up to $\mathrm{P}<0.01$ ). AMG641 significantly increased alkaline phosphatase activity and calcium phosphate deposition in both cell lines. Following treatment with AMG641 during osteogenic differentiation, in both cell lines CaSR expression was inversely related to that of osteogenic markers, as it was down-regulated in the large cell line and up-regulated in the small cell line. Inhibition of CaSR by NPS2390 blocked AMG641-related responses.

Conclusions: CaSR stimulation by extracellular calcium and AMG641-promoted in vitro osteogenic differentiation of equine UCMMSCs. The large and the small cell lines showed different behaviors that may be due to their different developmental and functional maturity [1]. CaSR activation may play a fundamental role in selecting specific differentiation checkpoints of this differentiation route, as related to MSC commitment status. These findings could be useful for downstream therapeutical applications

\section{References}

1. Martino NA, Lange-Consiglio A, Cremonesi F, Valentini L, Caira M, et al. (2011) Functional Expression of the Extracellular Calcium Sensing Receptor (CaSR) in Equine Umbilical Cord Matrix Size-Sieved Stem Cells. PLoS One. 6: 1-9. 


\section{Generation and Characterization of Human Cardiomyocytes Derived from Pluripotent Stem Cells}

Dell'Era $\mathbf{P}^{1}$, Benzoni $\mathbf{P}^{1}$, Crescini E', Serzanti $M^{1}$, Xia E', Campostrini $\mathbf{G}^{2}$ and Barbuti $A^{2}$

'FRU lab, Department Molecular and Translational Medicine, University of Brescia Health \& Wealth, Italy

${ }^{2}$ Department of Biosciences, University of Milan, Italy

In vitro development has been widely studied using murine ESCs (mESCs), whose differentiation procedure in culture implies the initial leukemia inhibitory factor (LIF) removal and the formation of cellular aggregates using the "hanging drop" method. These three dimensional (3D) structures, called embryoid bodies (EBs), replicate in vitro the different stages of murine embryonic development. Around differentiation day (dd) 8, clusters of spontaneously beating cells appear in culture; these cells express several transcriptional and structural cardiac markers and were therefore classified generically as cardiomyocytes. Since their isolation, human ESCs have shown different culturing needs from the murine counterpart, and their behavior revealed also minor differentiation plasticity. Cardiac differentiation is the most glaring example of this statement: only a modest proportion of EBs derived from either hESC or hiPSC contains contracting cells. This occurrence leads to the setup of different methods aimed to increase cardiac differentiation during in vitro development of pluripotent stem cells. While some of these procedures retain an initial 3D EB formation, others start from a confluent monolayer. However, CMs differentiated in vitro vary considerably from cells isolated from a mature human heart, because of the absence of humoral factors and organized mechanical and electrical stress. In general, many of the features of hPSC-CMs are reminiscent of normal fetal cells. hPSC-CMs are spontaneously beating cells co-expressing atrial-, ventricular-, and nodal- markers, with unorganized sarcomeres, immature mitochondria, and an expression profile different from adult CMs. The CMs that arise during early hESC or hiPSC in vitro differentiation exhibit spontaneous AP, with a relatively depolarized resting membrane potential, probably due to the temporary absence of the inward rectifier potassium current. The expression of the ion channels and, consequently, the ionic currents will undergo developmental maturation over time, as assessed by modifications in current density and property. hPSC-CMs immaturity is also reflected in their excitation-contraction machinery, lacking clear T-tubuli, disorganized sarcomeric striations, and immature $\mathrm{Ca}^{2+}$ handling.

Then the possibility to obtain human CMs in a culture dish is a powerful technique that will allow identification of new drugs in pharmacological studies as well as the identification of new causative genes in modeling genetic pathologies. Nevertheless, newly differentiated human CMs show a low degree of maturation that must be considered and, eventually, overcome by physical, mechanical or cultural stimuli. 


\title{
The Metabolically-Modulated Stem Cell Niche: An Energy Restriction Model for Cancer Stem Cell Resistance to Therapy
}

\author{
Bono S, De Oliveira Pagliaro S, Cheloni G, Tusa I, Lulli M, Rovida E and Dello Sbarba P
}

Dipartimento di Scienze Biomediche Sperimentali e Cliniche "Mario Serio", University of Florence - Istituto Toscano Tumori, Florence, Italy

The concept of stem cell niche was introduced in 1978 to model bone marrow sites suited to host hematopoietic stem cells (HSC) and favor their self-renewal, while restraining clonal expansion and commitment to differentiation. Studies of the effects of low oxygen tension on HSC maintenance in vitro led us to hypothesize, in 1993, that in vivo HSC niches are located within bone marrow (BM) areas where oxygen tension is physiologically lower than elsewhere. We then showed that HSC are capable to cycle in low oxygen, where, in turn, low oxygen steers cycling towards HSC self-renewal, pointing to a dynamic adaptation of HSC to metabolic restrictions.

Later, we addressed the question of whether metabolic restrictions (oxygen/glucose shortage) modulate the phenotype and resistance to therapy of cancer stem cells (CSC). We chose as a model disease chronic myeloid leukemia (CML), which is triggered by a well-identified oncogenetic mechanism (BCR/Abl expression) and brilliantly treated with tyrosine kinase inhibitors (TKi). However, TKi are extremely effective in inducing remission of disease, but unable, in most cases, to prevent relapse.

We found that metabolic restrictions, while suppressing BCR/Abl protein, do not abolish stem cell potential. This potential is therefore refractory to $\mathrm{TKi}$, pointing to a metabolic selection of cells responsible for TKi-resistant minimal residual disease (MRD) of CML and the related risk of relapse.

Studies of solid neoplasia brought another player into the game, low tissue $\mathrm{pH}$, which often parallels cancer growth and progression. Thus, a three-party scenario emerged for the regulation of CSC/MRD maintenance: the "hypoxic", the "ischemic" and the "acidic" niches, which are un likely constrained within rigid borders and are dynamically related to each other. 


\section{Regenerative Medicine}

\section{Enhanced Chondrogenic Potential of miR-221 and Slug depleted Human MSCs}

Lolli A', Lambertini E', Penolazzi L', Angelozzi $\mathbf{M}^{1}$, Narcisi ${ }^{2}$, van Osch GJVM² and Piva $\mathbf{R}^{1}$

'University of Ferrara, Department of Biomedical and Specialty Surgical Sciences, Italy

${ }^{2}$ Erasmus MC, Department of Orthopaedics, Rotterdam, Netherland

Human Mesenchymal Stromal Cells (hMSCs)-based tissue engineering is regarded as a very promising approach for cartilage regeneration. Our work is aimed at identifying new molecules having a crucial role in determining MSCs fate, and targeting such regulators for the guidance of chondrogenesis in the absence of differentiating agents, such as TGF- $\beta$. Recently, miR-221 and Slug transcription factor have emerged as anti-chondrogenic regulators. We investigated if the inhibition of these factors by specific antagomiR or siRNA molecule could be sufficient to address hMSCs towards chondrogenesis, in the absence of TGF- $\beta$, by conventional monolayer and three-dimensional (3D) culture. We demonstrated by immunocytochemistry assays that miR-221 or Slug silencing during monolayer culture increased the expression of the major cartilage protein Col2A1 and the master chondrogenic regulator Sox 9, while decreased CollA1 expression. Only Slug silenced hMSCs were able to increase the expression of TRPS1, a positive regulator of chondrocyte differentiation. Notably, Slug inhibition determined a reduction in the levels of miR-221, and we identified by chromatin immunoprecipitation (ChIP) assay a specific region of the miR-221 promoter that is involved in the in vivo recruitment of Slug. We showed that miR-221 silenced hMSCs cultured in pellet spontaneously undergo chondrogenesis, as demonstrated by a strong immunostaining for cartilage matrix proteins (Col2A1) and transcription factors (Sox9 and TRPS1) of the pellets at day 21. Finally, by embedding and culturing miR-221 or Slug depleted MSCs in alginate constructs, we confirmed the stability of the gene silencing for at least 28 days after combination with the scaffold. On-going experiments are aimed at evaluating the ability of the engineered hMSCs to effectively stimulate cartilage regeneration in vivo, by using an experimental model of osteochondral defect. Taken together, our findings demonstrate that miR-221 and Slug are functionally correlated in MSCs and that the silencing of these regulators is sufficient to induce differentiation towards the chondrogenic lineage, in the absence of TGF- $\beta$. Importantly, the combination of engineered hMSCs with a biocompatible scaffold preserved the efficiency of gene silencing, demonstrating the feasibility of this approach for the production of tissue engineering constructs that have the potential to regenerate articular cartilage. 


\section{The Secretome of Senescent Mesenchymal Stromal Cells Produces Different Outcomes on Normal and Cancer Cells}

Nicola Alessio², Stefania Capasso², Servet Ozcan ${ }^{3}$, Giovanni Di Bernardo ${ }^{2}$, Salvatore Cappabianca ${ }^{4}$, Marilena Cipollaro ${ }^{2}$ and Umberto Galderisi ${ }^{1-3}$

'Sbarro Institute for Cancer Research and Molecular Medicine, Center for Biotechnology, Temple University, Philadelphia, PA, USA

${ }^{2}$ Department of Experimental Medicine, Biotechnology and Molecular Biology Section, Second University of Naples, Naples, Italy

${ }^{3}$ Genome and Stem Cell Center (GENKOK), Erciyes University, Kayseri, Turkey

${ }^{4}$ Department "F. Magrassi - A. Lanzara" Second University of Naples, Naples, Italy

Cell senescence has been regarded as a strictly intracellular response, with the entire signaling circuitry, which takes place within the cell. Recent findings have demonstrated that several secreted molecules are associated with, and contribute to senescence proliferative arrest.

It is evident that senescence process may greatly affect also the composition of mesenchymal stem cells (MSC) secretome through a shift from a functional paracrine signaling to production of senescent-associated secreted factors that have potent autocrine and paracrine activities. Changes in secretome profiles of MSC may greatly impair their activities, which depend on the capability to secrete many factors, like cytokines and chemokines.

We performed a comparative analysis of human MSC secretome from young and replicative senescent cultures and evaluate if factors secreted from old MSC cultures may induce senescence, or arrest proliferation, or promote cytotoxic effects in young cells.

Our data strongly evidenced that senescence-associated secretory phenotype (SASP) implements a full senescence response in young cells suggesting that a few senescent cells in the MSC stem cell pool may be a potent trigger for ageing phenomena through a paracrine signaling cascade.

We demonstrated that secretion of IGFBP4 and IGBP7 has a significant senescent paracrine effect on young MSC. Moreover, the inhibition of these factors also reduced the percentage of apoptosis and promoted cell growth suggesting that may have a pleiotropic effect on MSC biology.

In conclusion, we could speculate that our study could pave the way to further investigations aiming to modify, in the near future, the current in vitro MSC expansion protocols for therapeutic purposes to avoid or reduce the occurrence of negative senescence related effects. 


\author{
New Insights into Donor-to-Donor Heterogeneity in Osteogenic Differentiation of h-MSCs: \\ Up-Regulation of Cystathionine-Beta-Synthase (CBS) and Cysthathionine-Gamma-Lyase (CTH/ \\ CSE) is A Selective Feature of Mineralizing h-MSCs \\ Gambari L', Gabusi E², Manferdini $C^{1}$, Paolella $F^{1}$, Piacentini $A^{1}$, Lisignoli $\mathbf{G}^{1}$ and Grassi $F^{2}$ \\ 'SC Laboratorio di Immunoreumatologia e Rigenerazione Tissutale, IOR, Bologna; 2Laboratorio RAMSES, IOR, Bologna, Italy
}

Background and Aims: A key limitation for the application of h-MSCs in bone regenerative medicine is their heterogeneous response to osteogenic stimuli. Both donor-to-donor and intra-population heterogeneity of MSCs has been widely described. However, to date no clear correlation has been identified between the expression profile of canonical osteogenic markers and functional heterogeneity of h-MSCs. The aim of our study was to investigate whether the expression profile of cystathionine-beta-synthase (CBS) and cysthathionine-gamma-lyase (CTH/CSE), the two key Hydrogen Sulphide $\left(\mathrm{H}_{2} \mathrm{~S}\right)$-producing enzymes is correlated with osteogenicity and could differentially identify the subset of h-MSCs which undergo osteogenic differentiation as opposed to those which do not display bone-forming ability.

Methods: h-MSCs were cultured in osteogenic medium and classified as mineralizing or non-mineralizing cells and non-, low, high- calcium deposing cells based on Alizarin Red (AR) staining quantification. Time-dependent and 'mineralization-dependent' modulation of CBS and CTH mRNA expression was evaluated with RT-PCR. Qualitative protein expression (immunohistochemistry and immunocytochemistry) and semi-quantitative mRNA and protein expression (RT-PCR and microarray, Western Blot) were performed to evaluate CBS and CSE/CTH in human bone tissue and/or h-MSCs and h-OBs isolated from same patients.

Results: CBS and CTH/CSE are expressed in human bone tissue as well as bone derived human cells. When subjected to osteogenic stimulation, $70 \%$ of h-MSCs undergo mineralization (with different levels of calcium deposition at each time point analyzed) and $30 \%$ remain unresponsive as revealed by AR staining. Time-dependent, significant up-regulation of CBS and CTH/CSE during osteogenesis is a selective feature of mineralizing h-MSCs ( $\mathrm{p}<0.0001$ and $\mathrm{p}<0,05$ respectively). Consistently, CBS and CTH/CSE are expressed ex-vivo at significantly higher levels in mature h-OBs as compared to h-MSCs obtained from same patients $(\mathrm{p}<0.001)$. Moreover, CBS expression is significantly correlated $\left(\mathrm{R}_{\mathrm{s}} 0.358 ; \mathrm{p}<0.0001\right)$ with the extent of mineralization in h-MSCs and is significantly up-regulated in h-MSCs progressing from a non-calcium deposing to a high-calcium deposing phenotype. Moreover, CBS expression level significantly discriminates mineralizing and non-mineralizing cells at day 14 and 21 during osteogenic culture $(\mathrm{p}<0.05)$.

Conclusions: CBS and CTH/CSE expression is unexpectedly associated with the commitment of h-MSCs to the osteoblastic cell lineage. Unlike canonical osteogenic markers, inducible up-regulation of these genes is a selective feature h-MSCs prone to osteogenic differentiation. These findings provide novel insights into the functional heterogeneity of h-MSCs and suggest that CBS and CTH/CSE expression can constitute a novel marker for the identification of the subset of mineralizing h-MSCs. 


\title{
How Can You Mend a Broken Heart (Bee Gees 1971)
}

\author{
Mauro Giacca
}

International Centre for Genetic Engineering and Biotechnology (ICGEB), Trieste, Italy

Fifteen million people worldwide are diagnosed of heart failure every year, mostly as a consequence of myocardial infarction or ischemic cardiomyopathy. Half of these patients are no longer alive after only 5 years from diagnosis. A vast part of this problems stems from the aging of the population, the incapacity of the heart to regenerate once damaged and the lack of novel drugs in the last 20 years. Thus, there is an impelling need to develop novel therapeutic strategies aimed at inducing cardiac repair and regeneration. In contrast to other species that regenerate the heart during the adult life, in mammals' damage to the myocardium is mended by a scarring mechanism. However, multiple evidences now indicate that a limited capacity of myocardial renewal also exists in adult individuals, and we are therefore actively searching for factors able to foster this regenerative capacity. Using viral vectors based on the Adeno-Associated Virus (AAV), which transduce the heart at very high efficiency, we are undertaking an exhaustive approach to identify extracellular proteins promoting cardiac repair, selected from an AAV library corresponding to the mouse secretome (1200+ secreted proteins). A second approach entails high throughput screening of microRNAs promoting cardiomyocyte proliferation. Starting from a whole genome human microRNA library, we have identified a few microRNAs endowed with the capacity of promoting expansion of cardiomyocytes in cell culture, inducing massive cardiac hyperplasty in the neonatal heart and improving cardiac function after myocardial infarction. These microRNA functions directly by activating the proliferative potential of differentiated cardiomyocytes, thus bypassing the requirement of stem cell expansion and differentiation. 


\author{
Microvesicle Secreted from Equine Amniotic Derived Cells and their Potential Role in In vitro Cell \\ Tendon Repair \\ Lange-Consiglio $A^{1}$, Perrini $C^{1}$, Tasquier $\mathbf{R}^{1}$, Deregibus $\mathrm{MC}^{2}$, Camussi $\mathrm{G}^{2}$, Pascucci $\mathrm{L}^{3}$, Marini $\mathrm{MG}^{4}$, Corradetti $\mathrm{B}^{4}$, Bizzaro $\mathrm{D}^{4}$ and $\mathrm{Cremonesi}^{1}$ \\ 'Università degli Studi di Milano, Italy \\ ${ }^{2}$ Centro di Biotecnologie Molecolari di Torino, Italy \\ ${ }^{3}$ Dipartimento di Medicina Veterinaria, Università degli Studi di Perugia, Italy \\ ${ }^{4}$ Università Politecnica delle Marche, Italy
}

Generally, the regenerative mechanisms ascribed to mesenchymal stem cells (MSCs) have been classified into 3 categories: differentiating toward reparative or replacement cell types, enhancing the nutrient supply, and improving the survival and function of the endogenous cells via paracrine actions. It is also known that, because of the inhospitable microenvironment of the injured or degenerating tissues, a large proportion of the implanted MSCs may die or undergo apoptosis in a short period post-transplantation, suggesting that mechanisms other than differentiation of transplanted cells could be present. This notion is supported by a recent study of Lange-Consiglio et al. [1] that injected AMC-conditioned medium (AMC-CM) in equine spontaneous injured tendons and ligaments with results consistent and overlapping (in terms of time interval needed to reach complete healing) with the beneficial effects of transplanted AMCs previously observed in the same animal model [2]. Administration of horse amniotic mesenchymal cells (AMCs) and their conditioned medium (CM) improved the in vivo recovery of spontaneous horse tendon lesions and inhibited the in vitro peripheral blood mononuclear cell proliferation. The process may involve paracrine factors but these factors and their mechanism to reach the target cells remain unknown. It has recently been demonstrated that extracellular vesicles or microvesicles (MVs) released from cells are an integral component of the cell-to-cell communication network involved in tissue regeneration [3] In the present study, the presence and the kind of MVs secreted by AMCs were investigated with NanoSight instrument and transmission electron microscopy. After this, the response of equine tendon cells to MVs was studied by a dose-response curve at different concentrations and different times and by a semi-quantitative analysis. Lastly, the ability of MVs to counteract an in vitro inflammatory process induced by lipopolysaccharide (LPS) on tendon cells was studied evaluating the expression of pro-inflammatory genes like metallopeptidasi (MPP) 1 and 13, and prostaglandin-endoperoxide synthase 2 (COX2). Results demonstrate that AMCs secrete MVs ranging in size from 100 to $1000 \mathrm{~nm}$ with a prevalence of 100-200 nm large MVs. Tendon cells were able to uptake them with an inverse relationship between concentration and time. The greatest incorporation was detectable at the concentration of $40 \times 10^{6} \mathrm{MVs} / \mathrm{ml}$ after $72 \mathrm{~h}$. MVs induced down-regulation of $M M P 1$ and $M M P 13$ expression, suggesting that they may have contributed along with soluble factors contained in $\mathrm{CM}$ to the in vivo regenerative effect on tendon lesions.

\title{
References
}

1. Lange-Consiglio A, Rossi D, Tassan S, Cremonesi F, Parolini O (2013) Conditioned medium from horse amniotic membrane-derived multipotent progenitor cells: immunomodulatory activity in vitro and first clinical application in tendon and ligament injuries in vivo. Stem Cells Dev. 22: 3015-3024.

2. Lange-Consiglio A, Tassan S, Corradetti B, Meucci A, Perego R, Bizzaro D, Cremonesi $F(2013)$ Investigating the potential of equine mesenchymal stem cells derived from amnion and bone marrow in equine tendon diseases treatment in vivo. Cytotherapy 10:1016-1023.

3. Camussi G, Deregibus MC, Bruno S, Cantaluppi V, Biancone L (2010) Exosomes/microvesicles as a mechanism of cell-to-cell communication. Kidney Int; 78: 838-848. 


\title{
Is Fundamental Adipose Derived Stem Cells Pre-Activation to Counteract Inflammation?
}

\author{
Manferdini $C^{1}$, Gabusi E'2, Paolella F', Piacentini $A^{1}$, Gambari $L^{1}$, Fleury-Cappellesso $S^{3}$, Barbero $A^{4}$ and Lisignoli $\mathbf{G}^{1}$ \\ 'SC Laboratorio di Immunoreumatologia e Rigenerazione Tissutale, IOR, Bologna,Italy \\ ${ }^{2}$ Laboratorio RAMSES, IOR, Bologna, Italy \\ ${ }^{3}$ EFS-Pyrénéés-Méditerranéé, Toulouse, France \\ ${ }^{4}$ Departments of Surgery and Biomedicine, University Hospital Basel, Basel, Switzerland
}

Adipose stromal cells (ASC) are promising candidate for cell therapy in osteoarthritis (OA), because they can have immunomodulatory, trophic and differentiation capacities. Synovial inflammation is now accepted as important feature for the symptoms and disease progression. Synovium is mainly composed of synovial fibroblast (SF) and macrophage (SM) and a low percentage of other cell types. Aim of the study was to analyze the role of SF and SM in counteracting the effects due to adipose stem cell co-culture.

Good manufacturing practices (GMP)-clinical grade ASC were isolated from subcutaneous adipose tissue. Synoviocytes were isolated from synovia of OA patients undergoing total joint replacement. Synoviocytes both at passage 1 (mainly composed of SM and SF) and 5 (only SF) were characterized for the following markers: CD3, CD11b, CD14, CD16, CD68, CD80, CD86, CD90, CD163, CD206, vimentin, VCAM-1, Collagen type 1 by flow cytometry, immunocytochemistry and q RT-PCR. Co-culture of synoviocytes p.1 and p.5 with ASC in transwell were performed. Macrophages type 1 (M1) and 2 (M2) were also isolated and co-cultured with ASC. Secreted inflammatory factors (IL6, CXCL8/IL8, CXCL1/GROa, CCL2/MCP-1, CCL3/MIP1 $\alpha$, CCL5/RANTES) were analyzed by multiplex bead-based sandwich immunoassay.

Synoviocytes at passage 1 were approximately $1 \%$ positive to CD3, $7 \%$ to CD14, $12 \%$ to CD16, $15 \%$ to CD68, $8 \%$ to CD80, 10\% to CD86, $13 \%$ to CD206 and 90\% to CD90 while synoviocytes at p. were negative to all the markers analyzed except for CD90, Vimentin, VCAM-1 and Collagen type 1. Moreover, synoviocytes at p.1 produced a significant higher amount of IL6, IL8/CXCL8, CCL2/MCP-1, CCL3/MIP1 $\alpha$ and CCL5/RANTES than at p.5. Interestingly, we confirmed that ASC reduced the release of inflammatory factors only when cultured with p.1 synoviocytes. By contrast, ASC co-cultured with p5 synoviocytes induced the release of all factors, except CCL3/MIP1 $\alpha$ that was still not released and expressed. Finally, we demonstrated that ASC effects were strictly dependent by macrophages type 1 and 2 that decreased the release of IL1 $\beta$, TNF $\alpha$ and CCL3/MIP1 $\alpha$.

These data demonstrate that the GMP-ASC effects on OA synovial inflammation is strictly dependent by macrophages. ASC counteracting effects mainly occur through released soluble factors by macrophage as well as by cells cross talk. 


\section{Osteogenic Differentiation of Amniotic Fluid Mesenchymal Stromal Cells and their Bone Regeneration Potential}

Caterina Pipino, Pamela Di Tomo, Domitilla Mandatori, Natalia Di Pietro, Sara Di Silvestre and Assunta Pandolfi

University "G. d'Annunzio", Chieti-Pescara, Italy; Aging Research Center, Ce.S.I. StemTeCh Group, 66013 Chieti, Italy

In orthopedics, tissue engineering approach using stem cells is a valid line of treatment for patients with bone defects. In this context, mesenchymal stromal cells of various origins have been extensively studied and continue to be a matter of debate. Although mesenchymal stromal cells from bone marrow are already clinically applied, recent evidence suggests that one may use mesenchymal stromal cells from extra-embryonic tissues, such as amniotic fluid, as an innovative and advantageous resource for bone regeneration [1]. The use of cells from amniotic fluid does not raise ethical problems and provides a sufficient number of cells without invasive procedures. Furthermore, they do not develop into teratomas when transplanted, a consequence observed with pluripotent stem cells. Their multipotent differentiation ability, low immunogenicity, and anti-inflammatory properties make them ideal candidates for bone regenerative medicine.

Notably, it has been recently demonstrated that cells derived from amniotic fluid are more easily reprogrammed into pluripotent stem cells than adult cells, with the advantage of using non-integrating systems, thus reinforcing their potential application in cellular replacement therapies $[2,3]$.

Indeed the established capacity of amniotic fluid cells to be rapidly reprogrammed offers the possibility of creating a bank of patientspecific pluripotent cells for pharmaceutical screening, disease modeling, and for potential applications in cellular replacement therapies in the orthopaedics field.

Today, various approaches have been applied to accelerate differentiation of amniotic fluid-derived cells into osteogenic cells and several studies have demonstrated how such osteoinductive molecules are able to get better this process. In this context, we recently demonstrated the role of calcimimetic drugs in improving the osteogenic differentiation of amniotic fluid cells, through activation of Calcium Sensing Receptor (CaSR), thus suggesting a strategy to develop a therapy against bone injury [4].

Therefore, the unique features of amniotic fluid-derived cells combined with the interesting results obtained in animal models of skeletal damage make them promising for a three-dimensional application of amniotic fluid cells in bone regeneration. Indeed, in vivo bone regeneration based on tissue engineering using scaffolds offers a plausible way of creating a favorable microenvironment for cells. Hence, various synthetic and non-synthetic scaffolds have been employed to support osteogenic differentiation of amniotic fluid cells.

These characteristics, together with the absence of ethical issues concerning their employment, suggest that amniotic fluid mesenchymal stromal cells might be promising candidates for tissue engineering and stem cell therapy of several human disorders. Therefore, a detailed understanding of the behavior of amniotic fluid mesenchymal stromal cells and their osteogenic ability is desirable considering a feasible application in bone regenerative medicine.

\section{References}

1. Pipino C, Pandolfi A (2015) Osteogenic differentiation of amniotic fluid mesenchymal stromal cells and their bone regeneration potential. World J Stem Cells 7: 681-690.

2. Moschidou D, Mukherjee S, Blundell MP, Drews K, Joneset GN, et al. (2012) Valproic Acid Confers Functional Pluripotency to Human Amniotic Fluid Stem Cells in a Transgene-free Approach. Mol Ther 20: 1953-1967.

3. Pipino C, Mukherjee S, David AL, Blundell MP, Shaw SW, et al. (2014) Trisomy 21 mid-trimester amniotic fluid induced pluripotent stem cells maintain genetic signatures during reprogramming: implications for disease modeling and cryobanking. Cell Reprogram. 16: 331-344.

4. Pipino C, Di Tomo $P$, Mandatori D, Cianci E, Lanuti $P$, et al. (2014) Calcium sensing receptor activation by calcimimetic $R-568$ in human amniotic fluid mesenchymal stem cells: correlation with osteogenic differentiation. Stem Cells Dev 23: 2959-2971. 


\section{Kidney Stem Cells and their Therapeutical Potential}

\section{Paola Romagnani}

Excellence Center DENOTHE, University of Florence and Meyer Children's Hospital, Florence, Italy

The kidney shares with other organs the ability to repopulate structures that have sustained some degree of injury. In the last few years, studies performed in the adult kidney supported the existence of renal progenitors with the potential to regenerate glomerular as well as tubular epithelial cells. Renal progenitors were first identified in human, where they localize within the Bowman's capsule as well as along the tubular compartment, and then also in mouse and in the zebra fish. One of the main outcome of the discovery of renal progenitors and of their function is that the kidney possesses an intrinsic potential of regeneration and functional repair of renal injury and consequently that prevention and treatment of end stage renal disease may be possible. However, this regenerative process is often inadequate because of abnormal or inefficient responses by renal progenitors. Indeed, very recent results suggest that renal progenitors can differentiate into podocytes and promote remission of glomerular disorders following podocyte injury. However, hyperplastic lesions that are frequently observed in patients affected by focal segmental glomerulosclerosis, collapsing glomerulopathy or crescentic glomerulonephritis, are also generated by proliferation of renal progenitors. These observations suggest that the outcome of renal disorders may depend from a balance between injury and regeneration provided by renal progenitors. Consistently, use of drugs that stimulate renal progenitors growth or differentiation exert protective effect on renal function and restore kidney tissue integrity. From all these findings it appears clear that understanding of how renal progenitors response to injury may be perturbed will be of crucial importance to set up novel treatments aimed at reversing kidney injury. 


\section{Regenerative Medicine}

\section{From Synchrotron Radiation to the most Recent Breakthroughs in Clinical Regenerative Dentistry}

Giuliani $A^{1}$, Langer $M^{2}$, Mangano $C^{3}$, Fiori $F^{1}$, Manescu $A^{1}$, Mazzoni $S^{1}$, Rustichelli $F^{1}$, Barone $A^{4}$, Mortellaro $C^{5}$, Piattelli $A^{6}$ and Papaccio $G^{7}$

Università Politecnica delle Marche - Dip. di Scienze Cliniche e Odontostomatologiche Via Brecce Bianche 1, Ancona, Italy

${ }^{2}$ Creatis, INSA-Lyon, Université CB Lyon 1 \& European Synchrotron Radiation Facility, France

${ }^{3}$ University of Insubria - Dept. of Morphological and Surgical Sciences, Varese, Italy

${ }^{4}$ Università di Pisa - Dipartimento di Chirurgia - Pisa, Italy

${ }^{5}$ Università del Piemonte Orientale - Dipartimento di Scienze Mediche, Novara, Italy

${ }^{6}$ University of Chieti - Department of Oral Medicine and Pathology, Chieti, Italy

${ }^{7}$ Secondo Ateneo di Napoli - Dipartimento di Medicina Sperimentale, Napoli, Italy

In recent years there has been an increasing interest in a novel approach to evaluate human bone biopsy specimens by means of synchrotron micro-tomography (SCT). Using SCT, bone regeneration subsequent to grafting hosting sites with different types of biomaterials (with or without stem cells seeding) is recently explored. Evaluation of the amount of bone formed is usually based on 2-D histomorphological data obtained from one or several histological sections.

If the regenerative potential of neighboring tissues with different morphology (alveolar process, unmineralized extracellular matrix involvement, regenerated vessels, etc.) on a defect or space to regenerate is not clearly verified or unknown, 3-D analyzing methods like high resolution SCT are indicated to explore the dynamic and spatial distribution of regenerative phenomena in such complex anatomic structures. Moreover the use of advanced techniques like Phase Contrast Tomography (PCT) and Holotomography (HT) allows visualizing components with low attenuation coefficient, like blood vessels.

In the present lecture the most recent breakthroughs in Clinical Regenerative Dentistry will be shown, demonstrating the unique capabilities of the SCT in offering not only an advanced characterization of different biomaterials (to understand the mechanism of their biological behavior as bone substitute) but also to investigate the growth kinetics of regenerated bone in different dental implants retrieved from humans.

Implant survival, bone regeneration, graft resorption, neo-vascularization and morphometric parameters (including anisotropy and connectivity index of the structures) were evaluated by microCT and HT at different times from implantation or grafting in human bone defects. 


\section{Potential Reparative Role of Resident Adult Renal Stem/Progenitor Cells (ARPCs) in Acute Kidney Injury}

Sallustio F, Serino G, Curci C and Schena FP

University of Bari "Aldo Moro", Italy

Human kidney is particularly susceptible to ischemia and toxins with consequential tubular necrosis and activation of inflammatory processes. This process can lead to the acute kidney injury (AKI) and even if the kidney has a great capacity for regeneration after tubular damage, in several circumstances the normal renal repair program may not be sufficient to achieve a successful regeneration. Recent studies have indicated that resident adult renal stem/progenitor cells could participate in this repair process and have the potentiality to enhance the renal regenerative mechanism. This could be achieved both directly, by means of their capacity to differentiate and integrate into the renal tissues, and by means of paracrine factors capable to induce or ameliorate the renal repair or regeneration.

Really, despite the several studies carried out on these renal progenitor cells, today there is still a debate in the field and the very existence of kidney epithelial stem cells remains an open question. In fact, some studies, published in the last two years, point out different theories. Again in 2014, another cell fate tracking study showed that proximal tubular cells, following different injuries, transiently acquire a "high regenerative" phenotype, with reparative characteristics. Their data suggested that do not exist a fixed progenitor cell population in the kidney.

In the same year, a third genetic lineage tracing study was performed, differently from the others, on long-term and unbiased clonal analysis regimen. It showed that, in the mouse kidney, progenitor cells exist as unipotent singly fated clones that continuously maintain and self-preserve the renal tissues throughout life. These precursors are activated by a WNT signal and, following kidney damage, regenerated new tubule segments through expansion of single clones that contributed to collective duct or proximal tubules.

Whether renal progenitors really exist and directly contribute to renal regeneration or not and therefore renal repair originate from dedifferentiated cells or cells acquiring a transient progenitor phenotype, the studies on the reparative characteristics of these "high regenerative" cells remain important.

We have shown that TLR2 may function as a sensor of the damage and its activation could led to the secretion of a series of chemokines useful for the renal repair and could stimulate the proliferation of tubular cells. Moreover, we found that progenitor cells are responsive to a fine regulation system in which BMP-2 mediates a negative feedback loop, balancing differentiation and proliferation by means of opposing effects on cell induction or proliferation. Another interesting finding about the renal progenitors is that aquaporins (AQP) 1,3 and 5 are expressed at both mRNA and protein levels in ARPCs. This observation led to the discovery of the presence of AQP5 in mammalian kidney, whose expression was previously unknown. Furthermore, another study showed that the retinoic acid promotes differentiation of ARPCs and protects against injury ameliorating the kidney function in multiple experimental models of AKI. Finally, a microengineered biochip resembling the structure of a kidney proximal tubule that embed ARPCs has been reported. The device allowed a recovery of urea, creatinine and glucose of $20 \%, 13 \%$ and $52 \%$, respectively. It could be further developed to investigate renal progenitors in vitro or to test drugs for their toxic effects. 


\section{Combined use of Mystem ${ }^{\mathrm{TM}}$, Micro- and Nano-Fat Methods to Improve Fat Graft Survival}

Giudice $\mathbf{G}^{1}$, Signorile $D^{1}$, Annoscia $\mathbf{P}^{1}$, Pugliese $D^{1}$ and Scacco $S^{2}$

'U.O.C di Chirurgia Plastica e Ricostruttiva Universitaria, Bari, Italy

${ }^{2}$ Dipartimento di Scienze Mediche di base, Neuroscienze e Organi di Senso , A.O.U. Policlinico di Bari, Bari, Italy

Introduction and Objectives: Fat grafting represents a promising treatment for soft tissues augmentation and regeneration. However it is affected by unpredictable results due to a large variability of graft survival rate (ranging from $40 \%$ to $80 \%$ ). This aspect becomes particularly relevant when a perfect symmetry is required. Recently, two new strategies have been proposed to overcome this limitation: a. reducing implanted adipose lobules size and $b$. increasing the number of adipose-derived progenitor cells (ADPCs) from the stromal vascular fraction (SVF) in the implant. To achieve these two goals, we propose a new protocol for the lipoaspirate processing which combines three methods.

Materials and Methods: Fat to be implanted was obtained from a lipoaspiration performed by a $2 \mathrm{~mm}$ blunt cannula, with dry technique, of a volume of fat about 2 times the amount needed for grafting. The total volume of lipoaspirate is divided in two equivalent portions, half to be used for lipotranfer (A) half to be processed (B) Portion A of the lipoaspirate is treated by MyStem ${ }^{\mathrm{TM}}$-Microfat, a mechanical fat fragmentation done using sterile microspheres. This fat is then submitted to a mechanic filtration by a microfluidic system $\left(\mathrm{MyStem}{ }^{\mathrm{TM}}\right)$. These devices allow us to separate the adipocytes from the liquid portion of the lipoaspirate, at the same time collecting the SVF-cells contained in that fraction. Portion B of lipoaspirate is treated by MyStem ${ }^{\mathrm{TM}}$-Nanofat, a shear-stress homogenizer that destroys all the adipocytes, while retaining the SVF and the extracellular matrix. Finally, the fat to be grafted in enriched with the ADPCs obtained from the total amount of fat $(\mathrm{A}+\mathrm{B})$.

Results: SVF-cells number has been assessed by direct cellular count on inverted-phase contrast microscope in a Burker glass chamber, with these results:

\begin{tabular}{|c|c|c|c|c|}
\hline Treatment & None (Control sample) & MyStem ${ }^{\mathrm{TM}}$ (Liquid portion) & Microfat \\
\hline SVF-cells Concentration & $6.1 \times 10^{6} / \mathrm{ml}$ & $7.1 \times 10^{6} / \mathrm{ml}$ & $8.4 \times 10^{6} / \mathrm{ml}$ \\
\hline SVF-cells $\%$ Increase & & $+16.4 \%$ & $17.2 \times 10^{6} / \mathrm{ml}$ \\
\hline
\end{tabular}

Conclusions: All the processing methods used in this study were able to increase SVF-cells concentration compared to unprocessed control fat sample. MyStem ${ }^{\mathrm{TM}}$ allowed us to collect SVF-cells from the liquid fraction of the lipoaspirate that is discarded in current methods. Nanofat resulted the best method to obtain a large number of SVF cells from the lobular stroma, at the same time eliminating all the adipocytes from the sample. For this reason it seems to be a very suitable method to enriching the fat to be implanted by SVF-cells, without affecting the fat graft volume. Microfat treatment increases the viability of the graft by creating smaller fat particles, consisting in small cluster of adipocytes. This allows a better diffusion of oxygen and nutrients from the hosting tissue to the fat graft. Moreover, the main advantage observed in the clinical use of this fat consists in its high-grade plasticity, allowing to use very thin injection cannulas. These new devices allow us to combine strategies, which could lead to relevant clinical advantages in the regenerative uses of lipotransfer techniques. Comparative clinical studies with traditional grafting techniques and adequate follow-up are requested to verify these hypotheses. 


\title{
Stem Cell Therapy in Muscular Dystrophies: State-of-the-Art and Future Perspectives
}

\author{
Meregalli $M^{1,2}$, Farini $A^{1}$, Belicchi $M^{1,2}$, Sitzia $C^{1}$, Razini $P^{1}$, Cassinelli $L^{1}$, Erratico $S^{2}$, Garcia $L^{3}$ and Torrente $Y^{1,2}$ \\ 'Stem Cell Laboratory, Department of Pathophysiology and Transplantation, Università degli Studi di Milano, Milan, Italy \\ ${ }^{2}$ Ystem S.r.I, Fondazione IRCCS Ca' Granda Ospedale Maggiore Policlinico di Milano, Centro Dino Ferrari, Milan, Italy \\ ${ }^{3}$ Institut de Myologie, Facultè de Medecine Pierre et Marie Curie, Paris, France
}

Muscular dystrophies are a heterogeneous group of inherited disorders presenting a large clinical variability regarding the age of onset, patterns of skeletal muscle involvement, heart damage, rate of progression and mode of inheritance. Attempts to repair muscle damage in Duchennes muscular dystrophy (DMD), the most severe case among muscular dystrophies, are facing several problems and no therapy is available yet.

Cell therapy is one promising approach to correct genetic diseases by contributing to tissue regeneration: stem cells can be isolated from a healthy donor or, when possible, from the same patient. In the first case cells will be transplanted under a regime of immune suppression while in the second case cells will have to be genetically corrected before transplantation in the same patient from which they were derived. The recent identification of different types of multi-potent stem cells, some of which are suitable for protocols of cell therapy, has disclosed new perspectives in the treatment of genetic diseases.

Our previous work indicated that CD133+ stem cells, a recently identified population of progenitor cells, produce functional improvement upon intra-arterial injection in a mouse model of muscular dystrophy.

Recently, transplantation of engineered dystrophic canine muscle-derived CD133+ cells has given promising results in Golden Retriever Muscular Dystrophy (GRMD) dogs, the most reliable animal model that shows a form of dystrophy very similar to DMD, which showed functional improvement after the treatment. We have therefore planned a pilot clinical trial, based on intra-muscular and intra-arterial transplantation of autologous engineered muscle-derived CD133+ cells. Efficacy and possible adverse effects will be evaluated to test whether this approach may represent a first step towards an efficacious therapy for muscular dystrophy. 